\title{
Potential Problems and Capacitance for a Conductor Bounded by Two Intersecting Spheres
}

\author{
By Chester Snow
}

\begin{abstract}
Formal expressions, as series and integrals, are derived for the external potential, which takes on assigned values on the two spherical boundary surfaces - either when the body is alone or when it is in any given axially symmetric electrostatic field. This is\} effected by representing a given function $f(x)$, for $1<x<\infty$, as a complex integral whose variable is the parameter $\mu$ of a Legendre function $P_{\mu-1 / 2}(x)$ or $Q_{\mu-1 / 2}(x)$.

The capacitance of the conductor is found as a series, whose terms involve psi-functions and vanish with $1 / n$ like $n^{-2}$. This is transformed into a formula depending upon a series whose $n^{\text {th }}$ term vanishes with $1 / n$ like $n^{-14}$.

In case the two spheres intersect at an angle $\omega$, which is a rational fraction of $2 \pi$, the capacitance is given in finite terms involving complete elliptic integrals, which is $Q_{-1 / 2}$.

The field, or electrostatic potential is given in finite terms when $\omega=n \pi / m$, where $m$ is any positive integer, and $n=1,2,3$, or 4 . The cases $n=3, n=4$ involve elliptic functions. These would permit the exact computation in finite terms of the penetration of an external applied field into a cavity with any angular aperture.
\end{abstract}

\section{Introduction}

This paper is concerned with the electrostatic field outside a body (in most cases a conductor) bounded by two intersecting spheres; some sections are shown in figure 1 . The potential has assigned values on its boundary (in most cases constant). The body may be isolated, or it may be in the presence of an external distribution of fixed charges that are axially symmetric.

To obtain the resulting potential distribution is therefore a question of finding the Green's function, this being the potential at any external point, $P$, when the boundary is kept at zero potential under the influence of any circular line-charge, $M^{\prime}$, which is coaxial with the axis of symmetry.

When the Green's function is found (as an integral or as an infinite series), the application to the conductor alone leads to expressions for its capacitance. There is also some physical interest in the evaluation of the electric field when the conductor is under external influence, such as a circular line-charge. This refers not only to the case of sections a, c, or d but also to those with cavities as in b, e, $f$ of figure 1 . In the latter, we may be interested in the amount of penetration of the external influence into the cavity, especially when the aperture is small.
The capacitance will be finite if one, but not both, of the intersecting spheres has infinite radius. The radius $a$ is taken as that which is always finite. This is on the right in all the case of figure 1. Passing to figure 2, a, the arc $A_{0} C$ of the meridian section has radius $a$. This sphere has the semiaperature $\theta$.

The angle, $\omega$, of intersection of the meridian ares is shown in figure 1 and also figure 2, a. This angle $\omega$ could have any value between zero and $2 \pi$, but it is evident from an inspection of the several cases in figure 1 that we may avoid a repetition of crescent-shaped sections, and with no loss of generality, by the restriction

$$
0<\theta<\pi \text { and } \theta<\omega<2 \pi \text {. }
$$

The radius $a_{1}$ of the second arc (fig. 2, a, $\operatorname{arc} A_{1} C$ ) is thus given by

$$
a_{1}=a \frac{\sin \theta}{|\sin (\omega-\theta)|} \cdot
$$

The capacitance will be a function of both angles, which may be denoted by $C_{\omega}(\theta)$.

To avoid getting lost in details that must be considered, we place here an outline of the main argument and the procedure we hope to follow. 


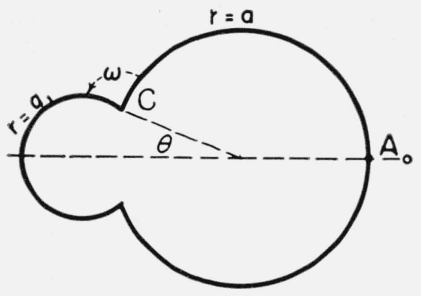

a
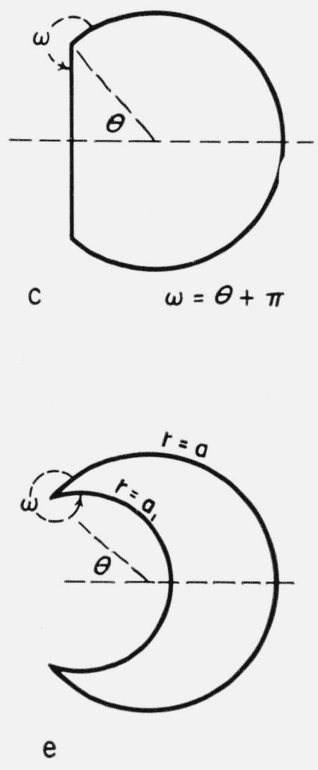

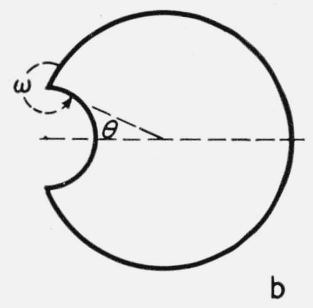

b

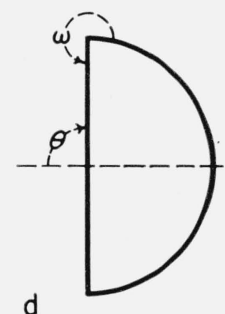

$\omega=\theta+\pi, \quad \theta=\frac{\pi}{2}$ (hemisphere)

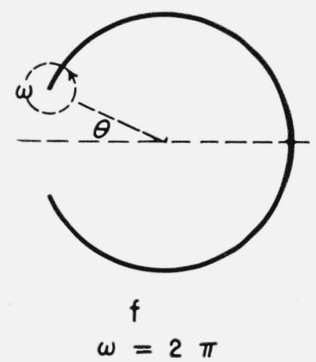

(thin shell)

Figure 1. Axial sections of conductors.

The cylindrical coordinates, $x, \rho$, will be used only in the initial picture, the $x$-axis being the axis of symmetry. As the conducting boundary is generated by revolution around the $x$-axis of the circular ares $A_{1} C$ and $A_{0} C$ of figure 2, a, we need only consider that half-plane of $z \equiv x+i \rho$ for which $\rho>0$. This half-plane, cut along the circular arc $A_{0} C$ (radius $a$ ), may be represented conformally upon the semi-infinite strip of the variable $\zeta=\alpha+\mathrm{i} \beta$, as indicated by similar letters in figure 2 , a and figure $2, \mathrm{~b}$. The equation of transformation is

$$
z=-\mathrm{c} \cdot \cot \left(\frac{\zeta-\theta}{2}\right)
$$

where

$$
c=a \sin \theta .
$$

The real equations giving $x$ and $\rho$ in terms of the toroidal coordinates $\alpha$ and $\beta$ are

and

$$
\begin{aligned}
& x=-c \cdot \frac{\sin (\alpha-\theta)}{\cosh \beta-\cos (\alpha-\theta)} \\
& \rho=c \frac{\sinh \beta}{\cosh \beta-\cos (\alpha-\theta)},
\end{aligned}
$$

and conversely,

$$
\cot (\alpha-\theta)=\frac{c^{2}-x^{2}-\rho^{2}}{2 c x}, \quad \operatorname{coth} \beta=\frac{c^{2}+x^{2}+\rho^{2}}{2 c \rho},
$$

$$
\sqrt{d x^{2}+d \rho^{2}}=c \frac{\sqrt{d \alpha^{2}+d \beta^{2}}}{\cosh \beta-\cos (\alpha-\theta)} .
$$

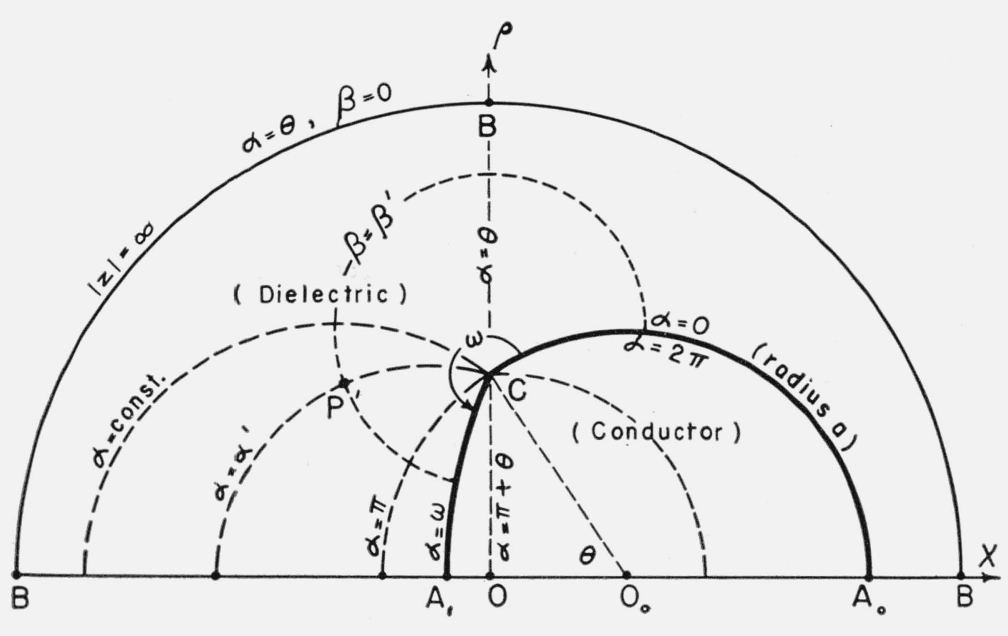

a

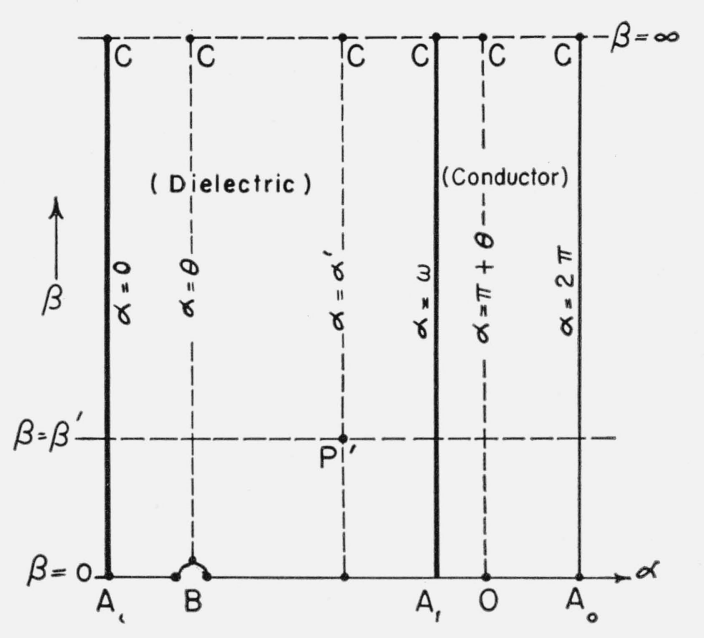

b

Figure 2.

a Half-plane of $z=x+i \rho$ cut along $\operatorname{arc} A_{0} C$. (Boundary of conductor is are $\alpha=0$, and $\alpha=\omega$ ); b, semi-infinite strip in plane of $\zeta=\alpha+i \beta$. 
The dotted circular arcs and the arcs bounding the meridian section of the conductor $(\alpha=0, \alpha=\omega)$ are parts of the locus of the equation ( $\alpha=$ constant)

$$
[x+c \cdot \cot (\alpha-\theta)]^{2}+\rho^{2}=\frac{c^{2}}{\sin ^{2}(\alpha-\theta)} .
$$

The family of circles $\beta=$ constant (orthogonal to the family $\alpha=$ constant) has the equation

$$
x^{2}+(\rho-c \cdot \operatorname{coth} \beta)^{2}=\frac{c^{2}}{\sinh ^{2} \beta} .
$$

Figure 2, a is drawn for the case in which $\pi<\omega<\pi$ $+\theta$.

When $x$ and $\rho$ are expressed in terms of $\alpha$ and $\beta$, the transform of any function $F(x, \rho)$ will be written $F(\alpha, \beta)$.

The dependent function most suitable for these coordinates is not the potential $V$ but the function $W$, where

$V(x, \rho)=V(\alpha, \beta)=\sqrt{\cosh \beta-\cos (\alpha-\theta)} W(\alpha, \beta)$

The potential equation,

$$
\nabla^{2} V=\left(D_{x}^{2}+D_{\rho}^{2}+\frac{1}{\rho} D_{\rho}\right) V=0
$$

transforms into

$$
\left(D_{\alpha}^{2}+D_{\beta}^{2}+\frac{1}{4 \sinh ^{2} \beta}\right) \sqrt{\sinh \beta} W=0
$$

or

$$
\left(D_{\alpha}^{2}+D_{\beta}^{2}+\operatorname{coth} \beta \cdot D_{\beta}+\frac{1}{4}\right) W=0,
$$

which has particular solutions,

$$
\begin{gathered}
W=\left(C_{1} \cos \nu \alpha+C_{2} \sin \nu \alpha\right) \cdot\left(C_{3} P_{\nu-1 / 2}(\cosh \beta)+\right. \\
\left.C_{4} Q_{\nu-1 / 2}(\cosh \beta)\right),
\end{gathered}
$$

where $C_{1}, C_{2}, C_{3}, C_{4}$ are arbitrary constants, $P$ and $Q$ being Legendre functions of $\cosh \beta$ of the first and second kind, respectively, with parameter $\nu-1 / 2$.

In a problem with $V$ given as a periodic function of $\alpha$ for $0<\alpha<2 \pi$ on the entire surface of a toroid, whose trace is some circle $\beta=\beta^{\prime}=$ constant, the function $W$ would thereby be a given periodic function of $\alpha$ on this circle. The parameter $\nu$ in particular solutions of the form (1.12) would therefore be determined by the requirement that $\cos \nu \alpha=\cos \nu(\alpha+2 \pi)$ and $\sin \nu \alpha=\sin \nu(\alpha+2 \pi)$, which require $\nu=0$, or an integer $n$. Hence de- velopment in a Fourier Series of the given function that represents $W(\alpha, \beta)$ is the key to the solution of such a problem.

However, the problem we have to consider is not completely analogous. The given function is here a function of $\beta$, for $0<\beta<\infty$, so that it resembles a case in which given functions of $\alpha$ must be represented by a Fourier integral. Normal functions do not exist, and the integral representations that will be required are not Fourier integrals.

In this problem the solution, $W$, must be constructed in the form of an integral with respect to ${ }_{\nu}$ of solutions like eq 1.12 , in the strip of figure $2, \mathrm{~b}$.

$$
0<\alpha<\omega \text { and } 0<\beta<\infty \text {, }
$$

which takes assigned values on the circular ares $\alpha=0$, and $\alpha=\omega$. To outline the method we may omit many details to be considered later. From here on we let

$$
x=\cosh \beta
$$

since we have no further need for the sumbol $x$ as a cylindrical coordinate. On the boundary are $A_{0} C(\alpha=0)$ of figure 2 , a,

$$
V(0, \beta)=V_{1}(x)=\sqrt{x-\cos \theta} W(0, \beta) .
$$

On the are $A_{1} C(\alpha=\omega)$,

$$
V(\omega, \beta)=V_{2}(x)=\sqrt{x-\cos (\omega-\theta)} W(\omega, \beta),
$$

where $V_{1}(x)$ and $V_{2}(x)$ are given functions of $x$ that do not become infinite at $C$ where $x=\infty$.

This assigns boundary values to $W$,

$$
\begin{gathered}
W(0, \beta)=f_{1}(x)=\frac{V_{1}(x)}{\sqrt{x-\cos \theta}}, \\
W(\omega, \beta)=f_{2}(x)=\frac{V_{2}(x)}{\sqrt{x-\cos (\omega-\theta)}},
\end{gathered}
$$

so $f_{1}(x)$ and $f_{2}(x)$ are given functions for $x$ for $1<x<\infty$, both vanishing like $x^{-1 / 2}$ when $x \rightarrow \infty$.

If a potential $V$ is required that is harmonic outside the body, vanishes at spatial infinity and takes the assigned values $V_{1}(x)$ and $V_{2}(x)$ on the boundary of the body, it might be expected to be given by an integral representing $W(\alpha, \beta)$, the integration being taken with respect to the parameter $\nu$. We may consider $\nu$ a complex variable $\nu=\nu_{1}+i \nu_{2}$ and imagine the integration to 
be taken along some abscissa $\nu_{1}=$ constant, in the $\nu$-plane.

We then consider a solution of eq 1.11 of the form

$$
\begin{gathered}
W(\alpha, \beta)=\frac{1}{\pi i} \int_{\nu_{1}-i \infty}^{\nu_{1}+i \infty} \frac{\nu P_{\nu-1 / 2}(x)}{\sin \nu \omega}\left[\sin \nu(\omega-\alpha) F_{1}(\nu)+\right. \\
\left.\quad \sin \nu \alpha F_{2}(\nu)\right] d \nu
\end{gathered}
$$

for $0 \leqslant \alpha \leqslant \omega$ and $1 \leqslant x \equiv \cosh \beta \leqslant \infty$.

This is an integral with respect to $\nu$ of particular solutions of eq 1.11 of the form of eq 1.12.

Spatial infinity in the plane figure 2 , a corresponds to the single point $\alpha=\theta, \beta=0$, in figure $2, \mathrm{~b}$. If this is an ordinary point, where $W$ remains finite, then the potential will vanish at spatial infinity as required, since the radical in eq 1.10 vanishes. At the boundary $\alpha=0$, the integral representing $W$ becomes (for $1 \leqslant x \leqslant \infty$ )

$W(0, \beta)=f_{1}(x)=\frac{1}{\pi i} \int_{\nu_{1}-i \infty}^{\nu_{1}+i \infty} \nu F_{1}(\nu) P_{\nu-1 / 2}(x) d \nu$.

And at the remaining boundary, $\alpha=\omega$,

$W(\omega, \beta)=f_{2}(x)=\frac{1}{\pi i} \int_{\nu_{1}-i \infty}^{\nu_{1}+i \infty} \nu F_{2}(\nu) P_{\nu-y_{2}}(x) d \nu$.

For each boundary arc the same problem is presented, of finding a function, $F(\nu)$, which is an analytic function of a complex variable $\nu=\nu_{1}+i \nu_{2}$ in some strip or half-plane of $\nu$, this function $F(\nu)$ being the solution of the integral equation.

$$
f(x)=\frac{1}{\pi i} \int_{\nu_{1}-i \infty}^{\nu_{1}+i \infty} \nu F(\nu) P_{\nu-1 / 2}(x) d \nu \text { for } 1<x<\infty,
$$

where $f(x)$ is an arbitrary, given function of $x$. The abscissa $\nu_{1}=R(\nu)=$ constant, which is the path of integration, must lie within limits to be found, which will depend in part upon the nature of the function $f(x)$ and in part upon the nature of the development-function $P_{\nu-1 / 2}(x)$. The solution of this integral equation is the subject of section III in which certain sufficient limitations upon $f(x)$ are found.

For this it is first necessary to place in section II some known properties of $P_{\nu-1 / 2}(x)$ and $\mathrm{Q}_{\nu-1 / 2}(x)$ as functions of $x$ and also of $\nu$. The things of especial importance for the integral representation (eq 1.17) are the principal terms of the asymptotic expansions of these two Legendre functions, both as to $x=\cosh \beta \rightarrow \infty$ ( $\nu$ fixed) and as to $\nu \rightarrow \infty \quad(x$ fixed).
After the integral representations are found in section III, the remainder of the paper consists in finding the Green's function in terms of a more fundamental function, $S$, and in exploiting this discovery for various applications. These applications are concerned with finding series more suitable in their convergence than that which first appears for computing capacitance. Also we find the cases in which the capacitance may be computed in finite terms. There are some cases in which the potential may be found in finite terms.

To outline this and to show the physical or geometrical role of the principal functions that appear, we may consider first the simple case in which the conductor is a complete sphere, the sphere $\omega=\pi$. In this case the arc $A_{1} C$ of figure 2, a $(\omega=\pi)$ is the continuation of the first circular are, $\alpha=0$, of radius $\alpha$.

The Green's function $V_{\pi}\left(\alpha, \beta ; \alpha^{1}, \beta^{1}\right)$ is defined as the potential at any point $P(\alpha, \beta)$ outside the sphere when there is a circular line-charge $M^{\prime}$ with trace at some fixed point $P^{\prime}\left(\alpha^{\prime}, \beta^{\prime}\right)$ also outside the sphere, while the sphere is kept at zero potential. The fixed point, $P^{\prime}$, is a point of singularity, for the potential becomes infinite when $P$ tends to coincide with $P^{\prime}$. It is the only one in the dielectric region (outside the sphere).

In case the line-charge $M^{\prime}$ shrinks to a pointcharge at some point $P^{\prime}\left(x^{\prime}, 0\right)$ or $P^{\prime}\left(\alpha^{\prime}, 0\right)$ on the $x$ axis, it is well known that the external potential (Green's function) at $P$ is equal to that of the actual point-charge at $P^{\prime}$ plus the potential at $P$ of a fictitious point-charge $M^{\prime \prime}$ within the sphere at a point $P^{\prime \prime}$. This point $P^{\prime \prime}$ is the image of $P^{\prime}$ by reflection in the sphere. The charge $M^{\prime \prime}$ must be opposite in sign to $M^{\prime}$ and suitably chosen proportional to $M^{\prime}$. (But $M^{\prime \prime} \neq-M^{\prime}$ as in the two-dimensional case of an endless circular cylinder.)

It will appear, and is fairly evident, that the same holds true for a circular line-charge, when (as we assume throughout) this is coaxial with the $x$ axis. Reference to eq 1.5 and to figure $2, \mathrm{~b}$ indicates that the image $P^{\prime \prime}\left(\alpha^{\prime \prime}, \beta^{\prime \prime}\right)$ of $P^{\prime}\left(\alpha^{\prime}, \beta^{\prime}\right)$ must be a point outside the strip $0<\alpha<\pi(=\omega)$, having the same height, that is, $\beta^{\prime \prime}=\beta^{\prime}$. Also in figure $2, \mathrm{~b}$, the points $P^{\prime}$ and $P^{\prime \prime}$ should be equidistant from the vertical line $A_{1} C$ of figure $2, \mathrm{~b}$, which represents part of the spherical boundary. By the same reasoning $P^{\prime}$ and $P^{\prime \prime}$ must be equidistant from the line $\alpha=0$ of figure 2, b. Hence 
there must be more than one image point $P^{\prime \prime}$ of $P{ }^{\prime}$ obviously an endless set of such image points to right and left, periodically distributed in the $\alpha \beta$-plane. However, all of these are represented on the $x, \rho$-plane by the single image point $P^{\prime \prime}$ inside the sphere.

As long as we are concerned only with a conductor in the shape of a complete sphere there is no sense in viewing the virtual charge at $P^{\prime \prime}$ as the superposition of an infinite number of charges. If we gradually change the boundary arc from $\omega=\pi$, this hidden structure makes its appearance and the innumerable images cannot in general be found within the conductor of figure 2 , a. The Green's function $V_{\omega}\left(\alpha, \beta ; \alpha^{\prime}, \beta^{\prime}\right)$ of the more general conductor $(\omega \neq \pi)$ cannot be expressed as the effect of any distribution of charges within the conductor of the $x, \rho$-plane.

The explanation may be found if we first extend the meaning of $V_{\pi}\left(\alpha, \beta ; \alpha^{\prime}, \beta^{\prime}\right)$ to the entire $x, \rho$-plane, in the sense that $P(\alpha, \beta)$ is the general point in the half-plane, while there are two fixed line charges one at $P^{\prime}$ the other at its image point $P^{\prime \prime}$. We may for the moment consider them both real and regard the spherical surface $(\alpha=0$ and $\alpha=\pi)$ merely as a surface where $V_{\pi}$ is zero. This half-plane is represented on any semi-infinite strip of $\alpha$-width $2 \pi$ in the $\alpha, \beta$-plane. Hence, extending its meaning still further, $V_{\pi}\left(\alpha, \beta ; \alpha^{\prime}, \beta^{\prime}\right)$ is a real periodic function of $\alpha$ with period $2 \pi$ having two singularities in any period interval such as $0<\alpha<2 \pi$.

As eq 1.10 suggests, it is simpler to consider the $W$-function as the Green's function, say $W_{\pi}(\alpha, \beta$; $\left.\alpha^{1}, \beta^{1}\right)$ for the differential eq 1.11. This also will be a periodic function of $\alpha$ with period $2 \pi$ having two singular points in a period interval.

From this it is a natural step to regard $W_{\pi}$ as the difference of two values of a simpler function $S_{\pi}\left(\alpha, \beta, \beta^{1}\right)$, which has period $2 \pi$ in $\alpha$ but only one singularity in a period.

For the sphere $\omega=\pi$, we find

$$
W_{\pi}\left(\alpha, \beta ; \alpha^{\prime}, \beta^{\prime}\right)=S_{\pi}\left(\alpha-\alpha^{\prime}, \beta, \beta^{\prime}\right)-S_{\pi}\left(\alpha+\alpha^{\prime}, \beta, \beta^{\prime}\right) .
$$

In case of a conductor bounded by two intersecting spheres the generalization is

$$
W_{\omega}\left(\alpha, \beta ; \alpha^{\prime}, \beta^{\prime}\right)=S_{\omega}\left(\alpha-\alpha^{\prime}, \beta, \beta^{\prime}\right)-S_{\omega}\left(\alpha+\alpha^{\prime}, \beta, \beta^{\prime}\right),
$$

where $S_{\omega}\left(\alpha, \beta, \beta^{\prime}\right)$ is the generalization of $S_{\pi}(\alpha, \beta$, $\left.\beta^{\prime}\right)$ and is the fundamental function for this problem. $S_{\omega}\left(\alpha, \beta, \beta^{\prime}\right)$ is an even periodic function of $\alpha$ with period $2 \omega$ having one singularity in any period interval such as $0<\alpha<2 \omega$.

The special function $S_{\pi}\left(\alpha-\alpha^{\prime}, \beta, \beta^{\prime}\right)$ introduced in section II, eq 2.25 , is in effect the $W$ function corresponding to the potential at $P(\alpha, \beta)$ produced only by the circular line charge with trace at $P^{\prime}\left(\alpha^{\prime}, \beta^{\prime}\right)$. It is, except for a simple but variable factor, a Legendre function $Q_{-1 / 2}$ with argument $\left[\cosh \beta \cosh \beta^{\prime}-\cos \left(\alpha-\alpha^{\prime}\right] / \sinh \beta \sinh \beta^{\prime}\right.$.

Its great practical advantage is that $S_{\pi}\left(\alpha, \beta, \beta^{\prime}\right)$ may be computed in terms of the complete elliptic integral $K$ for any value of its argument (eq 2.32). Unfortunately no finite expression has been found for the general function $S_{\omega}\left(\alpha, \beta, \beta^{\prime}\right)$.

The addition - theorem for $Q_{-1 / 2}$ gives two forms of Fourier's cosine series in $\alpha$ with coefficients that are products of Legendre functions of $\cosh \beta$ and $\cosh \beta^{\prime}$ (eq 2.26). The analogous series for $S_{\omega}$ is found in eq 4.9 .

As indicated in eq 1.15 to 1.17 the natural method of solving this problem will be through the use of integrals along some abscissa $\nu_{1}=$ constant in the plane of the complex variable $\nu=\nu_{1}+i \nu_{2}$. Anticipating this, we derive such an integral representing $S_{\pi}\left(\alpha, \beta, \beta^{\prime}\right)$. In section IV eq 4.6 is found, the similar integral representing its generalization $S_{\omega}\left(\alpha, \beta, \beta^{\prime}\right)$.

In section IV is derived the relation

$$
S_{\omega / m}\left(\alpha, \beta, \beta^{\prime}\right)=\sum_{t=0}^{m-1} S_{\omega}\left(\alpha+\frac{2 t \omega}{m}, \beta, \beta^{\prime}\right)
$$

where $m$ is a positive integer.

Out of this relation flow all the cases (rational values of $\omega / \pi$ ) for which the field outside the conductor may be found in finite terms. These are four infinite sets of values

$\omega=n \pi / m$ where $n=1,2,3$, or 4 , while with each $n$, the denominator $m$ may be any positive integer. The two sets with $n=3$ and $n=4$ involve elliptic functions. This is the subject of section IX.

On the other hand, it is found in section VIII that the capacitance is given in finite terms (with elementary functions) when $\omega=n \pi / m$, where $n$ and $m$ are any positive integers, subject to $0<\omega<2 \pi$.

In case $n$ or $m$ (or both) are large, these expressions may be less suitable for computation than some of the series in section VII, which apply for any $\omega$. In particular the form of eq 7.9 is a series 
of the form $\sum_{1}^{\infty} A_{n} \sin ^{2} n \pi \theta / \omega$ where the coefficients $A_{n}$ approach zero with increasing $n$ like $n^{-14}$.

In the sections that follow, we have not used the method of images as a guide. The results however may be interpreted from that point of view by an extension of the concept of images, in the course of which will appear the reason why the method of images has not been followed.

Interpreted on the endless $\alpha, \beta$-plane, the Green's function $V_{\pi}$ (or $W_{\pi}$ ), being periodic with period $2 \pi$, will of course have all its singularities endlessly repeated. The $x, \rho$-half-plane of figure 2 , a, which corresponds to actual space, is represented upon any semi-infinite strip of the $\alpha, \beta$-plane of $\alpha$-width $2 \pi$. But the inverse representation of the entire $\alpha, \beta$-plane upon the $x, \rho$-plane requires the latter to be imagined with infinitely many leaves lying above and below the plane of the paper of figure 2, a. Successive leaves are connected along the circular cut $A_{0} C$ of figure 2 , a, so that instead of crossing this cut we are forced up or down to another sheet.

All the singular points in $\alpha, \beta$-plane correspond to singular points on various sheets of the $x$, $\rho$-surface, all lying under or above the two points $P^{\prime}, P^{\prime \prime}$ of the first sheet, so it appears, looking down on them, as if there were only two.

For the general case $\omega \neq \pi$, the region of the $x_{0} \rho$-plane $0<\alpha<2 \omega$ (where $0<\omega<2 \pi$ ) is represented on any strip of the $\alpha, \beta$-plane of width $2 \omega$. Conversely, to represent the entire $\alpha$, $\beta$-plane on the $x \rho$-plane, this must be many-sheeted, with cuts at $\alpha=0, \alpha=2 n \omega$, where $n$ takes all integral values positive and negative. The endless number of singular points in the $\alpha \beta$-plane will correspond to an infinite distribution in the $x, \rho$-plane, of singular points that appear to be on the circle $\beta=\beta^{\prime}$, some inside the conductor and some outside apparently in the dielectric space where we placed only one. This one at $P^{\prime}$ is on the first sheet, and the others on different sheets but not hidden by $P^{\prime}$.

The potential produced at a point $P$ in the dielectric region of the $x \rho$-plane (the first sheet or plane of the paper in fig. 2, a), by a real or virtual circular line-charge with trace at $P^{\prime}$ also in the plane of the paper, is a comparatively simple expression given in section II. This expression becomes infinite when $P$ coincides with $P^{\prime}$ as it may without leaving the $x \rho$-sheet of actual space.
But if $P^{\prime}$ is in another sheet, then $P$ could come over it and cover it up, but the potential must remain finite and continuous since the singularity is on another sheet. Obviously the expression for the potential at $P$ is only to be found by an "analytic continuation" with respect to Laplace's equation, of the solution in one sheet due to singularity not on that sheet. If there were no alternative but the "method of images," we should have to wrestle with the questions of topology and find the "law of diminishing brightness" from one sheet to another. This way the method of images becomes a burden.

If it were not for this law of diminishing brightness, the entire circle $\beta=\beta^{\prime}$ in the $x \rho$-plane would appear continuously illuminated with singular points. Its effect would be to show a finite number of first magnitude (depending upon $\omega$ ). These would stand out among constellations and fainter nebulae. If at a turn of a lever the angle $\omega$ were gradually altered, there would be certain rational values of $\omega / \pi$, say $\omega=n \pi / m$, for which the kaleidoscopic view clears up. The luminous background disappears and there remains only a finite number of singular points on this circle. This corresponds to eq 1.19 , in which $S_{\omega}\left(\alpha, \beta \beta^{\prime}\right)$ may be evaluated in finite terms. Even then some of these images must be considered anomalous, as they appear to be in the dielectric region on the circle $\beta=\beta^{\prime}$.

\section{Preliminary Equations}

Those known relations between Legendre functions that will be required are placed in this section for reference. Also the known expression, in the coordinates $\alpha, \beta$, for the potential of a circular linecharge, whose generalization is fundamental to the problem, is presented here.

Since $x$ is a favorite notation for a real variable and there is no further use of the rectangular coordinate $x$, there should be no confusion if from here on we let $x=\cosh \beta$. The two functions, $y=$ $Q_{\nu-1 / 2}(x)$ and $y=Q_{-\nu-1 / 2}(x)$ are solutions of Legendre's equation

$$
\frac{d}{d x}\left[\left(x^{2}-1\right) y^{1}(x)\right]=\left(\nu^{2}-\frac{1}{4}\right) y(x),
$$

where for $1<x<\infty$,

$$
Q_{\nu-1 / 2}(x) \equiv \frac{\Gamma\left(\frac{1}{2}\right) \Gamma\left(\frac{1}{2}+\nu\right)}{(2 x)^{1 / 2+\nu} \Gamma(1+\nu)} F\left(\frac{1}{4}+\frac{\nu}{2} \frac{3}{4}+\frac{\nu}{2}, 1+\nu ; \frac{1}{x^{2}}\right)
$$


Considering $0<\beta<\infty$, that is, $1<x<\infty$, it is evident that $Q_{\nu-1 / 2}(x) / \Gamma\left(\frac{1}{2}+\nu\right)$ is an integral function of the complex variable $\nu=\nu_{1}+i \nu_{2}$, so that $Q_{\nu-1 / 2}(x)$ is an analytic function of $\nu$, whose only singularities in the finite $\nu$-plane are on the negative real axis where

$$
\nu=\nu_{1}+i 0=-\left(n+\frac{1}{2}\right) \text { where }(n=0 \quad 1,2,3, \ldots) .
$$

These are simple poles of the factor $\Gamma\left(\frac{1}{2}+\nu\right)$. The other factor, $1 / \Gamma(1+\nu)$, vanishes when $\nu=-n$, but eq 2.2 shows that

$$
Q_{-n-1 / 2}(x)=Q_{n-1 / 2}(x),
$$

and $Q_{n-1 / 2}(x)$ is a finite and continuous function of $x$ for $1<x<\infty$.

For all other values of $\nu$ the two solutions, $Q_{\nu-1 / 2}(x)$ and $Q_{-\nu-1 / 2}(x)$, are linearly independent as shown by the identity,

$$
\begin{gathered}
\left(x^{2}-1\right)\left[Q_{\nu-1 / 2}(x) Q_{-\nu-1 / 2}^{\prime}(x)-Q_{\nu-1 / 2}^{\prime}(x) \cdot Q_{-\nu-1 / 2}(x)\right] \\
=\pi \tan \nu \pi .
\end{gathered}
$$

For general values of $x$ and $\nu$, the solution $P_{\nu-1 / 2}$ $(x)$ of eq 2.1 may be defined by

$P_{\nu-1 / 2}(x) \equiv P_{-\nu-1 / 2}(x) \equiv-\frac{\cot \nu \pi}{\pi}\left[Q_{\nu-1 / 2}(x)-Q_{-\nu-1 / 2}(x)\right]$.

Whenever $Q_{\nu-1 / 2}$ exists it is linearly independent of $P_{\nu-1 / 2}$, as shown by the identity derived from eq 2.3,

$$
\left(x^{2}-1\right)\left[Q_{\nu-1 / 2}(x) P_{\nu-1 / 2}^{\prime}(x)-Q_{\nu-1 / 2}^{\prime}(x) P_{\nu-1 / 2}(x)\right]=1 .
$$

Considering $x$ as a constant real parameter greater than one, it is evident that the definition (eq 2.4) makes $P_{\nu-1 / 2}(x)$ an even integral function of $\nu$; for the poles of $\cot \nu \pi$ at $\nu=n$ are removed by the vanishing of the bracket. Also the poles of either $Q$-function at $\nu=-\frac{1}{2} \pm n$ are removed by the vanishing of $\cot \nu \pi$.

Using eq 2.2 in eq 2.4 gives, for $1<x<\infty$,

$$
\begin{aligned}
P_{\nu-1 / 2}(x)=- & \frac{\cot \nu \pi}{\sqrt{2 \pi x}}\left[\frac{\Gamma\left(\frac{1}{2}+\nu\right)}{(2 x) \nu \Gamma(1+\nu)} F\left(\frac{1}{4}+\frac{\nu}{2}, \frac{3}{4}+\frac{\nu}{2}, 1+\nu ; x^{-2}\right)-\right. \\
& \left.\frac{\Gamma\left(\frac{1}{2}-\nu\right)}{(2 x)^{-\nu} \Gamma(1-\nu)} F\left(\frac{1}{4}-\frac{\nu}{2}, \frac{3}{4}-\frac{\nu}{2}, 1-\nu ; x^{-2}\right)\right] .
\end{aligned}
$$

From analytic continuations of the hypergeometric functions in eq 2.2 and 2.6 we obtain, for $0<\beta<\infty$,

$$
Q_{\nu-1 / 2}(x)=\sum_{s=0}^{\infty} \frac{\Gamma\left(s+\frac{1}{2}\right) \Gamma\left(s+\nu+\frac{1}{2}\right)}{\Gamma(s+1) \Gamma(s+\nu+1)} e^{-(2 s+\nu+1 / 2) \beta}=\frac{e^{-(\nu+1 / 2) \beta} \Gamma\left(\frac{1}{2}\right) \Gamma\left(\nu+\frac{1}{2}\right)}{\Gamma(1+\mu)} F\left(\frac{1}{2}, \frac{1}{2}+\nu, 1+\nu ; e^{-2 \beta}\right)
$$

and

$$
P_{\nu-1 / 2}(x)=e^{-(\nu+1 / 2) \beta} F\left(\frac{1}{2}, \frac{1}{2}+\nu, 1 ; 1-e^{-2 \beta}\right),
$$

whence

$$
P_{-1}(x) \equiv P_{0}(x) \equiv 1 \text {. }
$$

$$
P_{\nu-1 / 2}(x)=\cosh \beta^{-(\nu+1 / 2)} F\left(\frac{1}{4}+\frac{\nu}{2}, \frac{3}{4}+\frac{\nu}{2}, 1 ; \tanh ^{2} \beta\right)=\frac{\cosh \beta^{-(\nu+1 / 2)}}{\Gamma\left(\nu+\frac{1}{2}\right)} \sum_{s=0}^{\infty}\left(\frac{\tanh \beta}{2}\right)^{2 s} \frac{\Gamma\left(2 s+\nu+\frac{1}{2}\right)}{\Gamma^{2}(s+1)},
$$

and

$Q_{\nu-1 / 2}(x)=P_{\nu-1 / 3}(x) \log (2 \operatorname{coth} \beta)-$

$$
\frac{\cosh \beta^{-(1 / 2+\nu)}}{\Gamma\left(\frac{1}{2}+\nu\right)} \sum_{s=0}^{\infty}\left(\frac{\tanh \beta}{2}\right)^{2 s} \frac{\Gamma\left(2 s+\nu+\frac{1}{2}\right)}{\Gamma^{2}(s+1)}\left[\psi\left(2 s+\nu+\frac{1}{2}\right)-\psi(s+1)\right]
$$


where

$$
\psi(z) \equiv \Gamma^{\prime}(z) / \Gamma(z) .
$$

For the manipulation of certain integrals we require only the principal term in the asymptotic expansion of these functions. This is needed in two senses, the one when $x \rightarrow \infty$ (with $\nu$ constant) and the other when $\nu \rightarrow \infty$ (with $x$ constant). From eq 2.2 and 2.6 it may be shown that

When $x=\cosh \beta \rightarrow \infty$,

$$
\left.\begin{array}{rl}
Q_{\nu-1 / 2} \rightarrow 0 & \text { if } \nu_{1}>-\frac{1}{2}(\text { or } \nu=-1,-2,-3, \ldots) \\
& \rightarrow \infty \text { if } \nu_{1}<-\frac{1}{2}(\text { or } \nu \neq-1,-2, \ldots . . .)
\end{array}\right\} .
$$

Hence

$$
Q_{\nu-1 / 2} \rightarrow 0 \text {, and } Q_{-\nu-1 / 2} \rightarrow 0 \text { if }-\frac{1}{2}<\nu_{1}<\frac{1}{2} .
$$

Consequently,

$$
\left.\begin{array}{rl}
P_{\nu-1 / 2} & \rightarrow 0 \text { if }-\frac{1}{2}<\nu_{1}<\frac{1}{2}\left(\text { with any } \nu_{2}\right) \\
& \rightarrow \infty \text { if } \frac{1}{2}<\left|\nu_{1}\right|\left(\text { with any } \nu_{2}\right)
\end{array}\right\}
$$

(If $\nu_{2}=0$ and $\nu_{1}= \pm \frac{1}{2}, P_{0} \equiv P_{-1} \equiv 1$ ).

Let $\lambda=\lambda_{1}+i \lambda_{2}$ and $\nu=\nu_{1}+i \nu_{2}$.

By eq 2.1 and the corresponding equation with $\nu=\lambda$, we find, by use of eq 2.10 and 2.11 , that if $\nu_{1}>-\frac{1}{2}$

$$
\begin{aligned}
& \int_{1}^{\infty} Q_{\nu-1 / 2}(x) Q_{\lambda-1 / 2}(x) d x= \\
& \frac{\psi\left(\nu+\frac{1}{2}\right)-\psi\left(\lambda+\frac{1}{2}\right)}{\nu^{2}-\lambda^{2}} \text { if } \lambda_{1}>-\nu_{1},
\end{aligned}
$$

whence

$$
\begin{aligned}
& \int_{1}^{\infty} Q_{-\nu-1 / 2}(x) Q_{\lambda-1 / 2}(x) d x= \\
& \frac{\psi\left(-\nu+\frac{1}{2}\right)-\psi\left(\lambda+\frac{1}{2}\right)}{\nu^{2}-\lambda^{2}} \text { if } \lambda_{1}>\nu_{1} .
\end{aligned}
$$

From this, by use of eq 2.4 and the identity

$$
\psi\left(\nu+\frac{1}{2}\right)-\psi\left(-\nu+\frac{1}{2}\right)=\pi \tan \nu \pi,
$$

it follows that

$$
\int_{1}^{\infty} P_{\nu-1 / 2}(x) Q_{\lambda-1 / 2}(x) d x=\frac{1}{\lambda^{2}-\nu^{2}} \text { if }\left|\nu_{1}\right|<\lambda_{1} .
$$

If the conditions stated are not satisfied, each of these three integrals diverges. If they are, each converges absolutely because of the exponential factor arising from $\exp \left[-\left(\nu+\frac{1}{2}\right) \beta\right]$ in eq 2.7.

In the second sense we consider $\beta$ fixed

$$
0<\beta<\infty \text {. }
$$

When $\nu=\nu_{1}+i \nu_{2} \rightarrow \infty$, there is the known asymptotic term.

$$
Q_{\nu-1 / 2}(\cosh \beta) \sim \sqrt{\frac{\pi}{2 \sinh \beta}} \frac{e^{-\nu \beta}}{\sqrt{\nu}},
$$

which becomes zero or infinity according as $\nu_{1} \geqslant 0$ or $\nu_{1}<0$. This also applies when $\nu \rightarrow$ along the negative real axis of $\nu$ without passing through the poles of $Q_{\nu-1 / 2}$ at $\nu=-\left(n+\frac{1}{2}\right)$.

Whence

$$
Q_{-\nu-1 / 2}(\cosh \beta) \sim \mp i \sqrt{\frac{\pi}{2 \sinh \beta}} \frac{e^{\nu \beta}}{\sqrt{\nu}},
$$

and by eq 2.4 ,

$$
P_{\nu-1 / 2}(\cosh \beta) \sim \frac{e \nu^{\beta} \pm i e^{-} \nu^{\beta}}{\sqrt{2 \pi^{\nu} \sinh \beta}}
$$

(where upper sign is for ${ }_{2}>0$, lower for ${ }^{\nu}<0$.

Whence $P_{\nu-1 / 2}$ always becomes infinite when the real part of $\nu$ (i. e. $\nu_{1}$ ) becomes $\pm \infty$.

On the other hand if $\nu_{2} \rightarrow \pm \infty$ while $\nu_{1}$ remains finite, each of these functions vanishes, and in such a manner that, when $1<x<\infty$, the integrals along any finite abscissa $\nu_{1}$.

$$
\left.\begin{array}{l}
\int_{\nu_{1}-i \infty}^{\nu_{1}+i \infty} \nu F(\nu) P_{\nu-1 / 2}(x) d \nu \text { and } \\
\int_{\nu_{1}-i \infty}^{\nu_{1}+i \infty} \nu F(\nu) Q_{\nu-1 / 2}(x) d \nu \\
\text { rge if, and only if, } \\
\nu^{1 / 2} F(\nu) \rightarrow 0 \text { when } \nu_{2} \rightarrow \pm \infty .
\end{array}\right\}
$$

will converge if, and only if, 
Another relation that will be required may be derived from

$$
Q_{\nu-1 / 2}(\cosh \beta)=\int_{-1}^{1} \frac{\left(1-t^{2}\right)^{\nu-1 / 2} d t}{[2(\cosh \beta-t)]^{\nu+1 / 2}} \text { for }\left(\begin{array}{c}
0<\beta \\
-1 / 2<\nu_{1}
\end{array}\right) \text {. }
$$

This is, in effect, Schlafli's integral, and is shown to be equivalent to eq 2.7 by expansion of the integral. If this is written

$$
\int_{-1}^{e^{-\beta}}(\quad) d t+\int_{e^{-\beta}}^{1}(\quad) d t
$$

and the variable of integration $t$ is replaced by $\beta^{\prime}$ where $2 e^{-\beta^{\prime}}=\left(1-t^{2}\right) /(\cosh \beta-t)$ in both, but

$$
t=e^{-\beta^{\prime}}-\sqrt{1-2 e^{-\beta^{\prime}}} \cosh \beta+e^{-\sqrt[2]{\beta^{\prime}}}
$$

in the 1st integral, and

$$
t=e^{-\beta^{\prime}}+\sqrt{ }
$$

in the 2nd integral, it is found that the two in-

tegrals are equal, so that

$$
Q_{\nu-1 / 2}(\cosh \beta)=\frac{1}{\sqrt{2}} \int_{\beta}^{\infty} \frac{e^{-\nu \beta^{\prime}} d \beta^{\prime}}{\sqrt{\cosh \beta^{\prime}-\cosh \beta}} .
$$

For reference, the appropriate expressions may be placed here that represent the potential at any point $P(x, \rho)$ or $P(\alpha, \beta)$, which is due only to a circular line-charge $M^{\prime}$ with trace at $\left.P^{\prime}\left(x^{\prime}\right) \rho^{\prime}\right)$, (i.e. $\left.P^{\prime}\left(\alpha^{\prime}, \beta^{\prime}\right)\right)$.

It may be written $V_{\pi}\left(\alpha, \beta ; \alpha^{\prime}, \beta^{\prime}\right)=\frac{M^{\prime}}{2 c}\{[\cosh$ $\left.\beta-\cos (\alpha-\theta)]\left[\cosh \beta^{\prime}-\cos \left(\alpha^{\prime}-\theta\right)\right]\right\}^{3 / 2} S_{\pi}\left(\alpha-\alpha^{\prime}, \beta\right.$, $\left.\beta^{\prime}\right)$.

where $S \pi$ is never negative and defined by

$$
\begin{aligned}
& S_{\pi}\left(\alpha, \beta, \beta^{\prime}\right) \equiv \frac{2}{\pi}\left[\sinh \beta \sinh \beta^{\prime}\right]^{-1 / 2} Q_{-1 / 2} \\
& \left(\frac{\cosh \beta \cosh \beta^{\prime}-\cos \alpha}{\sinh \beta \sinh \beta^{\prime}}\right) .
\end{aligned}
$$

The so-called addition-theorem for this function is

$$
\begin{aligned}
S_{\pi}\left(\alpha, \beta ; \alpha^{\prime}, \beta^{\prime}\right) & =\frac{4}{\pi} \sum_{n=0}^{\infty} \epsilon_{n} Q_{n-1 / 2}\left(\cosh \beta^{\prime}\right) P_{n-1 / 2}(\cosh \beta) \cos n \alpha \text { if } 0 \leqslant \beta \leqslant \beta^{\prime} \\
& =\frac{4}{\pi} \sum_{0}^{\infty} \epsilon_{n} Q_{n-1 / 2}(\cosh \beta) P_{n-1 / 2}\left(\cosh \beta^{\prime}\right) \cos n \alpha \text { if } 0 \leqslant \beta^{\prime} \leqslant \beta,
\end{aligned}
$$

where $\epsilon_{0}=1 / 2 \epsilon_{n}=1$ for $n>0$. The series converges when $\beta=\beta$,' provided that $\cos \alpha \neq 1$.

When $\beta^{\prime} \rightarrow 0$, the line-charge shrinks to a pointcharge on the $x$-axis, and this becomes

$$
\begin{aligned}
S_{\pi}(\alpha, \beta, 0) & =\frac{\sqrt{2}}{\sqrt{\cosh \beta-\cos \alpha}} \\
& =\frac{4}{\pi} \sum_{0}^{\infty} \epsilon_{m} Q_{m-1 / 2}(\cosh \beta) \cos m \alpha,
\end{aligned}
$$

which converges for all values of $\alpha$ when $0<\beta<\infty$, but not when $\beta=0$, since each coefficient $Q_{n-1 / 2}$ then becomes infinite. However,

$$
S_{\pi}(\alpha, 0,0)=\frac{1}{\left|\sin \frac{\alpha}{2}\right|},
$$

since the radical is always positive in eq 2.27 .

The preceding series are useful, but we may now derive an integral representing $S_{\pi}\left(\alpha, \beta, \beta^{\prime}\right)$ more useful and comprehensive. Both forms of series (eq 2.26) may be derived from it: also it converges even when $\beta=\beta^{\prime}=0$.

$$
S_{\pi}\left(\alpha, \beta, \beta^{1}\right)=\frac{1}{i} \int_{\nu_{1}-i \infty}^{\nu_{1}+i \infty} \frac{\cos \nu(\pi-|\alpha|)}{\cos \nu \pi} P_{\nu-1 / 2}(\cosh \beta) P_{\nu-1 / 2}\left(\cosh \beta^{1}\right) d \nu,
$$

where

To prove this, first take the path along $\nu_{1}=$ $-\frac{1}{2}<\nu_{1}<\frac{1}{2}$ and $\left(\begin{array}{l}0 \leqslant|\alpha| \leqslant 2 \pi \text { when } \beta \neq \beta^{\prime} \\ 0<|\alpha|<2 \pi \text { when } \beta=\beta^{\prime}\end{array}\right)$. constant where $0<\nu_{1}<\frac{1}{2}$ and replace $P_{\nu-1 / 2}(\cosh \beta)$ by the second member of eq. 2.4, which gives

$$
\begin{aligned}
& S_{\pi}\left(\alpha, \beta, \beta^{1}\right)=-\frac{1}{\pi i} \int_{\nu_{1}-i \infty}^{\nu_{1}+i \infty} \frac{\cos \nu(\pi-|\alpha|)}{\sin \nu \pi} Q_{\nu-1 / 2}(\cosh \beta) P_{\nu-1 / 2}\left(\cosh \beta^{\prime}\right) d \nu+ \\
& \frac{1}{\pi i} \int_{\nu_{1}-i \infty}^{\nu_{1}+i \infty} \frac{\cos \nu(\pi-|\alpha|)}{\sin \nu \pi} Q_{-\nu-1 / 2}(\cosh \beta) P_{\nu-1 / 2}\left(\cosh \beta^{\prime}\right) d \nu .
\end{aligned}
$$

The substitution $\nu=-\nu^{\prime}$ in the 2 nd integral gives (since $P_{-\nu-1 / 2} \equiv P_{\nu-1 / 2}$ )

$$
\left.I_{2}=\frac{-1}{\pi i} \int_{-\nu_{1}-i \infty}^{-\nu_{1}+i \infty} \frac{\cos \nu(\pi-|\alpha|)}{\sin \nu \pi} Q_{\nu-1 / 3} \cosh \beta\right) P_{\nu-1 / 2}\left(\cosh \beta^{\prime}\right) d \nu .
$$


This path may be translated from the abscissa $-\nu_{1}$, to that, $+\nu_{1}>0$, of the original path, taking into account the contribution to $I_{2}$ of amount $\frac{2}{\pi} Q_{-1 / 2}(\cosh \beta) P_{-1 / 2} \cosh \beta^{\prime}$ of the pole at $\nu=0$.

Hence if $0<|\alpha|<2 \pi$, eq. 2.29 is equivalent to

$$
S_{\pi}\left(\alpha, \beta, \beta^{\prime}\right)=\frac{2}{\pi} Q_{-1 / 2}(\cosh \beta) P_{-1 / 2}\left(\cosh \beta^{\prime}\right)-\frac{2}{\pi i} \int_{\nu_{1}-i \infty}^{\nu_{1}+i \infty} \frac{\cos \nu(\pi-|\alpha|)}{\sin \nu \pi} Q_{\nu-1 / 2}(\cosh \beta) P_{\nu-1 / 2}\left(\cosh \beta^{\prime}\right) d \nu
$$

where $0<\nu_{1}<1$.

This is valid when $\beta$ and $\beta^{\prime}$ are interchanged.

When $\beta^{\prime}<\beta$, the asymptotic expressions (eq 2.18 and 2.20) show that this path may be closed with an infinite semicircle on which $\nu_{1}>0$. It then encircles (in a positive trigonometric direction) the poles at $\nu=n=1,2,3, \ldots$ where $\sin \nu \pi=0$. The sum of the residues gives the series (2.26).

The special case $\beta^{\prime}=0$ of eq 2.29 gives on reference to eq 2.27 the integral representation,

$$
\begin{gathered}
S_{\pi}(\alpha, \beta, 0)=\frac{\sqrt{2}}{\sqrt{\cosh \beta-\cos \alpha}}= \\
\frac{1}{i} \int_{\nu_{1}-i \infty}^{\nu_{1}+i \infty} \frac{\cos \nu(\pi-|\alpha|)}{\cos \nu \pi} P_{\nu-1 / 2}(\cosh \beta) d \nu,
\end{gathered}
$$

where $-\frac{1}{2}<\nu_{1}<\frac{1}{2}$ and $\left(\begin{array}{l}0 \leqslant \alpha \leqslant 2 \pi \text { if } \beta \neq 0 \\ 0<\alpha<2 \pi \text { if } \beta=0\end{array}\right)$.

It may be noticed that the value of $S_{\pi}\left(\alpha, \beta, \beta^{\prime}\right)$ may be found from tables of the complete elliptic integral $K$ with modulus $k$. Equation 2.7 shows that

$$
Q_{-1 / 2}(x)=2 \sqrt{k} K(k)
$$

where $k=x-\sqrt{x^{2}-1}=e^{-\beta}$ when $x=\cosh \beta$.

\section{Integral Representations of a Given Function}

We may now take up the problem formulated in eq 1.17. Let $f(x)$ be a function of the real variable, $x=\cosh \beta$, for $1<x<\infty$, which may be given arbitrarily in this interval except for certain restrictions to be found. Consider the problem of finding the function $F(\nu)$ of the complex variable $\nu=\nu_{1}+i \nu_{2}$, which is the solution of the integral equation,

$f(x)=\frac{1}{\pi i} \int_{\nu_{1}-i \infty}^{\nu_{1}+i \infty} \nu F(\nu) P_{\nu-1 / 2}(x) d \nu$ for $1<x<\infty$,

where an essential part of the hypothesis for determining $F(\nu)$ is that this equation holds when the path of the integral is along any abscissa $\nu_{1}=$ constant, which lies in some half-plane, to be found, say

$$
-\frac{1}{2} \leqslant \nu_{0}<\nu_{1}<\infty \text { and }-\infty<\nu_{2}<\infty .
$$

This implies that

$\nu F(\nu)$ is analytic in this half-plane.

It also implies that $F(\nu)$ vanishes in a certain manner when $\nu \rightarrow \infty$ in any direction lying in this half-plane.

In case $f(x) \rightarrow \infty$ when $x \rightarrow 1$, the integral (eq $3.1 \mathrm{a}$ ) is only required to converge for $1<x<\infty$. In that case, as shown in eq 2.21 , it is necessary that

$$
\nu^{1 / 2} F(\nu) \rightarrow 0 \text { when } \nu \rightarrow \infty .
$$

In case $f(1)$ is finite, the convergence of eq $3.1 \mathrm{a}$ for $x=1$, (where $P_{y-1 / 2}(x) \equiv 1$ ) requires

$$
\nu^{2} F(\nu) \text { is bounded when } \nu \rightarrow \infty \text {. }
$$

This condition shows the convergence of $\int_{p} \nu F(\nu) d \nu$, where the path $p$ is an infinite semicircle that closes the path. Since by eq 3.2a $\int_{\nu_{1}-i \infty}^{\nu_{1}+i \infty} \nu F(\nu) d \nu+\int_{p} \nu F(\nu) d \nu=0$, the first integral converges.

Let

$$
G(\lambda) \equiv \int_{1}^{\infty} f(x) Q_{\lambda-1 / 2}(x) d x \text { where } \lambda=\lambda_{1}+i \lambda_{2} .
$$

Assume for the moment that restrictions on the nature of $f(x)$ may be found that are sufficient to make $G(\lambda)$ an analytic function of $\lambda$ in the halfplane $\nu_{0}<\lambda_{1}$ and to make $G(\lambda)$ vanish like $F(\nu)$ as specified in (3.2 a, b, c). ( $\nu_{0}$ is to be found). Take a positive abscissa $a=\nu_{0}+\epsilon$, if $0<\nu_{0}$, or $a=\epsilon$ when $\nu_{0} \leqslant 0$ where $\epsilon$ is a positive constant, arbitrarily small. 
Taking $\nu_{1}=a$ in eq 3.1a and replacing $f(x)$ in the integral defining $G(\lambda)$ by the integral (eq 3.1 a) gives

$$
G(\lambda)=\frac{1}{\pi i} \int_{1}^{\infty} Q_{\lambda-1 / 2}(x) d x \int_{a-i \infty}^{a+i \infty} \nu F(\nu) P_{\nu-1 / 2}(x) d \nu .
$$

We may prove that $F(\nu)=G(\nu)$ in the half-plane $0<a \leqslant \nu_{1}$, and the conditions (eq 3.2) will then be sufficient (in those cases where $\nu_{0}<0$ ) for the continuation of the equation $F(\nu)=G(\nu)$ down to this negative $\nu_{0}$.

The validity of interchange of order of integration may be secured by taking $\lambda_{1}>a$,

$$
G(\lambda)=\frac{1}{\pi i} \int_{a-i \infty}^{a+i \infty} \nu F(\nu) d \nu \int_{1}^{\infty} Q_{\lambda-1 / 2}(x) P_{\nu-1 / 2}(x) d x .
$$

Since $\lambda_{1}>a=\nu_{1}>0$, we have by eq 2.17 the absolutely convergent integral,

$$
\int_{1}^{\infty} Q_{\lambda-1 / 2}(x) P_{\nu-1 / 2}(x) d x=-\frac{1}{\nu^{2}-\lambda^{2}},
$$

so that

$G(\lambda)=-\frac{1}{2 \pi i} \int_{a-i \infty}^{a+i \infty} \frac{2 \nu F(\nu)}{\nu^{2}-\lambda^{2}} d \nu$, and by eq 3.2 or or $3.2 \mathrm{c}$, this converges absolutely so the inversion of order is permissible. Also, since $F(\nu)$ vanishes at $\nu \rightarrow \infty$, this path may be closed by an infinite semicircle on which $\nu_{1} \geqslant a>0$. It then encircles in positive direction the single pole at $\nu=\lambda$. Consequently by Cauchy's theorem $G(\lambda)=F(\lambda)$ when $a<\lambda_{1}<\infty$, that is, $F(\nu)=G(\nu)$ for $a<\nu_{1}<\infty$.

Therefore, when eq 3.1a holds for the halfplane (eq $3.1 \mathrm{~b}$ ), its solution is

$$
F(\nu)=\int_{1}^{\infty} f(x) Q_{\nu-1 / 2}(x) d x .
$$

In that case $f(x)$ has the integral representation,

$$
f(x)=\frac{1}{\pi i} \int_{\nu_{1}-i \infty}^{\nu_{1}+i \infty} \nu P_{\nu-1 / 2}(x) d \nu \int_{1}^{\infty} f\left(x^{\prime}\right) Q_{\nu-1 / 2}\left(x^{\prime}\right) d x^{\prime},
$$

for $1<x<\infty$,

where the path is along any abscissa $\nu_{1}$, in the halfplane $\nu_{0}<\nu_{1}$. The value of $\nu_{0}$ depends in part upon the nature of $f(x)$ when $x \rightarrow \infty$, see eq 3.17 below. In case $\nu_{0}$ is negative this is equivalent to

$$
f(x)=\frac{1}{\pi i} \int_{\nu_{1}-i \infty}^{\nu_{1}+i \infty} \nu Q_{\nu-1 / 2}(x) d \nu \int_{1}^{\infty} f\left(x^{\prime}\right) P_{\nu-1 / 2}\left(x^{\prime}\right) d x^{\prime},
$$

where the path is along any abscissa $\nu_{1}$ in the strip

$$
\nu_{0}<\nu_{1}<\left|\nu_{0}\right| \text { where } \nu_{0}<0 .
$$

Relation (3.5 a) is derived by noting that the function

$$
F(-\nu)=\int_{1}^{\infty} f(x) Q_{-\nu-1 / 2}(x) d x \text { is analytic where }
$$

$-\infty<\nu_{1}<\left|\nu_{0}\right|$. Hence, when, and only when, $\nu_{0}<0$, there exists a strip in which $F(\nu)$ and $F(-\nu)$ are each analytic. In that case, $P_{\nu-1 / 2}(x)$ in eq 3.4 may be replaced by its definition in eq. 2.4. In the resulting integral with $Q_{-\nu-1 / 2}$, make the substitution $\nu=-\nu^{\prime}$ and then translate the path to the original. This gives eq 3.5a. If we let $\nu=-\nu^{\prime}$ in eq $3.5 \mathrm{a}$ and then recover the path by translation, this gives

$f(x)=-\frac{1}{\pi i} \int_{\nu_{1}-i \infty}^{\nu_{1}+i \infty} \nu Q_{-\nu-1 / 2}(x) d v \int_{1}^{\infty} f\left(x^{\prime}\right) P_{\nu-1 / 2}\left(x^{\prime}\right) d x^{\prime}$,

$$
\text { where } \nu_{0}<\nu_{1}<\left|\nu_{0}\right|
$$

Taking halif the sum of eq 3.5a and $3.5 \mathrm{~b}$ gives, by use of eq 2.4,

$$
\begin{aligned}
f(x)=-\frac{1}{2 i} \int_{\nu_{1}-i \infty}^{\nu_{1}-i \infty} \nu \tan \pi & P_{\nu-1 / 2}(x) d \nu \\
& \int_{1}^{\infty} f\left(x^{\prime}\right) P_{\nu-1 / 2}\left(x^{\prime}\right) d x^{\prime},
\end{aligned}
$$

where $\nu_{0}<\nu_{1}<\left|\nu_{0}\right|$.

The last three representations of $f(x)$, eq $3.5 \mathrm{a}, \mathrm{b}$, c, are of limited application compared to the first in eq 3.4 since they require that $\nu_{0}$ be negative, the imaginary axis $\nu_{1}=0$, being than a possible path. It is evident that they apply along the imaginary axis when $e^{\beta / 2} f(\cosh \beta) \rightarrow 0$ as $\beta \rightarrow \infty$ if $f(x)$ satisfies the other conditions to be given in eq $3.6 \mathrm{a}$ and $3.6 \mathrm{~b}$ below, for in that case the integral $\int_{1}^{\infty} f(x) P_{\nu-1 / 3}(x) d x$ will converge.

When $f(x)$ satisfies certain sufficient conditions to be given in eq 3.6, the sense in which this function is represented by eq 3.4 may be indicated as follows:

For this let

$$
I\left(x, x_{0}, \eta\right) \equiv \frac{1}{\pi i} \int_{\nu_{1}-\eta}^{\nu_{1}+i \nu} \nu P_{\nu-1 / 2}(x) d \nu \int_{1}^{x_{0}} f\left(x^{\prime}\right) Q_{\nu-1 / 2}\left(x^{\prime}\right) d x^{\prime}
$$

and

$$
\phi(x)=(\underset{\eta \rightarrow \infty}{\operatorname{limit}})\left(\operatorname{limit}_{x_{0} \rightarrow \infty}\right) I\left(x, x_{0}, \eta\right) .
$$


If $x$ is a point at which $f(x)$ is not infinite, we may prove that $\phi(x)=[f(x+0)+f(x-0)] / 2$ or $f(x)$ if this is a value of $x$ where $f(x)$ is continuous.

As before it is sufficient to take $0<a \leqslant \nu_{1}<\infty$.
The limit $x_{0} \rightarrow \infty$ of the $x^{\prime}$-integral is the transform $F(\nu)$ of $f(x)(3.3)$, and this integral converges absolutely and also uniformily as to $\nu_{2}$ in any finite interval $-\eta<\nu_{2}<\nu$. Hence taking $\nu_{1}=a>0$,

$$
\begin{aligned}
\phi(x)= & \left(\lim _{x_{0} \rightarrow \infty}\right)\left(\lim _{\eta \rightarrow \infty}\right) \frac{1}{\pi i} \int_{1}^{x_{0}} f\left(x^{\prime}\right) d x^{\prime} \int_{a-i \eta}^{a+i \eta} P_{\nu-1 / 2}(x) Q_{\nu-1 / 2}\left(x^{\prime}\right) d \nu=\left(\lim _{x_{0} \rightarrow \infty}\right)\left(\lim _{\eta 0 \rightarrow \infty}\right) \frac{1}{\pi i} \int_{0}^{\beta_{0}} \sinh \beta^{\prime} f\left(\cosh \beta^{\prime}\right) d \beta^{\prime} \\
& \int_{a-i \eta}^{a+i \eta} \nu P_{\nu-1 / 2}(\cosh \beta) Q_{\nu-1 / 2}\left(\cosh \beta^{\prime}\right) d \nu,
\end{aligned}
$$

where $x=\cosh \beta$ and $x_{0}=\cosh \beta_{0}$.

It is here assumed that the constant $x$ is a value for which the assigned function $f(x)$ is not infinite, but also not necessarily continuous.

In the $\nu$-integral the integrand is an analytic function of $\nu$, that is, without singularity at any finite point for which $-\frac{1}{2}<\nu_{1}$. Hence the integral from the point $a-i \eta$ to $a+i \eta$ (where $a>0$ ) may be taken along any path between these terminal points, which lies to the right of them. Taking the path as a semicircle of radius $\eta$, we may then take $\eta$ so large that the integral will differ by an arbitrarily small amount from that which is obtained by use of the asymptotic expansions (eq 2.19 and 2.20). This is

Hence

$$
\phi(x)=\left(\lim _{\boldsymbol{\beta}_{0} \rightarrow \infty}\right)\left(\lim _{\eta \rightarrow \infty}\right) \frac{1}{\pi} \int_{0}^{\beta_{0}} e^{a\left(\beta-\beta^{\prime}\right)} \sqrt{\frac{\sinh \beta^{\prime}}{\sinh \beta}} f\left(\cosh \beta^{\prime}\right) \frac{\sin \eta\left(\beta-\beta^{\prime}\right)}{\beta-\beta^{\prime}} d \beta^{\prime} .
$$

Assuming (3.6 a, b, c) below, and that $f(x)$ is of limited variation in any finite interval, this limit becomes, by Dirichlet's theorem,

$$
\begin{aligned}
\phi(x) & =\frac{1}{2}[f(\cosh \beta+0)+f(\cosh \beta-0)] \\
& =\frac{1}{2}[f(x+0)+f(x-0)],
\end{aligned}
$$

which is the meaning of eq 3.4 .

The following restrictions upon $f(x)$ are sufficient to make its transform, $F(v)$, satisfy the conditions (eq $3.2 \mathrm{a}, \mathrm{b}, \mathrm{c}$ ) and to define the limiting abscissa $\nu_{0}$.

In the interval $1<x<\infty$ there may be at most a finite number of points $x_{1}, x_{2}, \ldots$ where $f(x)$ has finite or infinite discontinuities. None of these points are limit-points, that is, $f(x)$ is of limited variation in any finite interval. All are such that

$$
\int_{1}^{x}|f(x)| d x \text { converges for any finite } x .
$$

There is a real constant $\delta$ (which may be positive, negative, or zero) such that when $x=$ cosh $\beta \rightarrow \infty$,

$$
f(x) \sim C(x) x^{-\delta}=C(\beta) e^{-\delta \beta},
$$

where $C(x)$ is bounded. Similarly, when $x \rightarrow 1$,

$$
f(x) \sim C_{0}(x)(x-1)^{-\delta_{0}}=C_{0}(\beta)\left(1-e^{-2 \beta}\right)^{-2 \delta_{0}}
$$

where

$$
\delta_{0}<\frac{3}{4}
$$

or

$$
f(x) \sim C_{0} \log (x-1)
$$

$\left(C_{0}(\mathrm{x})\right.$ bounded $)$.

Reference to eq 2.7 shows that the limiting abscissa of the half-plane, in which $F(\nu)$ is analytic, is the algebraically greater of the two

$$
\nu_{0}=\frac{1}{2}-\delta \text { or } \nu_{0}=-\frac{1}{2} \text {. }
$$

The proof of this, that is, the derivation of the three conditions (eq 3.2a,b,c from eq 3.6a,b,c), (for $\nu_{0}$ defined in eq 3.7 ), may be made by investigating the series obtained by use of eq 2.7, which is

$$
\begin{aligned}
& F(\nu) \equiv \int_{1}^{\infty} f(x) Q_{\nu-1 / 2}(x) d x=\int_{0}^{\infty} \sinh \beta f(\beta) Q_{\nu-1 / 2} \\
& (\cosh \beta) d \beta=\frac{1}{2} \sum_{s=0}^{\infty} \frac{\Gamma\left(s+\frac{1}{2}\right) \Gamma\left(s+\nu+\frac{1}{2}\right)}{\Gamma(s+1) \Gamma(s+\nu+1)} \\
& \int_{0}^{\infty} e^{-(2 s+\nu-1 / 2) \beta}\left(1-e^{-2 \beta}\right) f(\cosh \beta) d \beta .
\end{aligned}
$$


When $\beta$ is large the most important term is the first $(s=0)$, and it is evident that the convergence of this integral at the upper limit is secured (whatever the value of $\delta$ ) by taking $\nu_{1}>\nu_{0}$ where $\nu_{0}$ is defined in eq 3.7. This will make $F(\nu)$ analytic in the hali-plane $\nu_{1}>\nu_{0}$ as required in eq $3.2 \mathrm{a}$.

For the remaining conditions $(3.2 \mathrm{~b}, \mathrm{c})$ we may first consider the case where the only singularities of $f(x)$ are at $x=1$ and $x=\infty$. For this purpose take for comparison the function

$$
f_{1}(x)=f_{1}(\cosh \beta)=e^{-\delta \beta}\left(1-e^{-2 \beta}\right)^{-2 \delta_{0}},
$$

whose transform is

$$
F_{1}(\nu)=\frac{\Gamma(\gamma)}{4} \sum_{s=0}^{\infty} \frac{\Gamma\left(s+\frac{1}{2}\right) \Gamma\left(s+\nu+\frac{1}{2}\right) \Gamma\left(s+\frac{1}{2}\left[\nu+\delta-\frac{1}{2}\right]\right)}{\Gamma(s+1) \Gamma(s+\nu+1) \Gamma\left(s+\frac{1}{2}\left[\nu+\delta-\frac{1}{2}\right]+\gamma\right)}
$$

where $\gamma \equiv 2\left(1-\delta_{0}\right)$. Since $\gamma$ is positive this series converges absolutely in the half-plane and we may show that $\nu^{2-2 \delta_{0}} F(\nu)$ is bounded when $\nu \rightarrow \infty$. (3.9c) For eq $3.2 \mathrm{~b}$ we have $2-2 \delta_{0}>\frac{1}{2}$ since $\delta_{0}<\frac{3}{4}$. For eq $3.2 \mathrm{c}$ when $\delta_{0}=0, f(1)$ finite, $\nu^{2} F(\nu)$ is bounded. Since a positive constant $M$ could be found such

$$
|F(\nu)|<M\left|F_{1}(\nu)\right|
$$$$
F_{1}(\nu) \sim \frac{\Gamma(\gamma)}{4} \sum_{s=0}^{\infty} \frac{\Gamma\left(s+\frac{1}{2}\right)}{\Gamma(s+1)\left(s+\nu+\frac{1}{2}\right)^{\frac{1}{2}}\left(s+\frac{1}{2}\left[\nu+\frac{1}{2}\right]\right)^{\frac{1}{2}+\gamma}},
$$

this would suffice when eq $3.9 \mathrm{c}$ is proved.

To prove this we have

$$
\frac{\Gamma(z+\alpha)}{\Gamma(z+\gamma)} \sim z^{\alpha-\nu}\left[1+\text { zero }\left(\frac{1}{z}\right)\right] \text { when } z \text { is large. }
$$

Hence in the series of eq $3.9 \mathrm{~b}, \nu$ may be taken so large that the series will differ by any preassigned small fraction of itself from

that is,

$$
C_{2}\left|\sum_{0}^{\infty} \frac{\Gamma\left(s+\frac{1}{2}\right)}{\Gamma(s+1)\left(s+\nu+\frac{1}{2}\right)^{\frac{1}{2}+\gamma}}\right|<\frac{4\left|F_{1}(\nu)\right|}{\Gamma(\gamma)}<C_{1} \mid \sum_{0}^{\infty} \frac{\Gamma\left(s+\frac{1}{2}\right)}{\Gamma(s+1)\left(s+\frac{1}{2}\left[\nu+\frac{1}{2}\right]\right)^{\frac{1}{2}+\gamma}},
$$

$$
C_{2}\left|H\left(\nu+\frac{1}{2}\right)\right|<\frac{4\left|F_{1}(\nu)\right|}{\Gamma(\gamma)}<C_{1} H\left(\frac{1}{2}\left[\nu+\frac{1}{2}\right]\right),
$$

where $C_{1}$ and $C_{2}$ are positive constants, and

$$
H(\nu)=\left[\frac{\sqrt{\pi \Gamma(\gamma)}}{\Gamma\left(\gamma+\frac{1}{2}\right)}\right] \frac{\Gamma(\nu)}{\Gamma(\nu+\gamma)}=\frac{\sqrt{\pi \Gamma(\nu)}}{\Gamma\left(\nu+\gamma+\frac{1}{2}\right)} F\left(\frac{1}{2}, \nu, \nu+\gamma+\frac{1}{2} ; 1\right) .
$$

When $\nu$ is large this becomes

$$
H(\nu) \sim\left[\frac{\sqrt{\pi \Gamma(\gamma)}}{\Gamma\left(\gamma+\frac{1}{2}\right)}\right] \frac{1}{\nu^{2}} .
$$

Replacing $\nu$ successively by $\nu+\frac{1}{2}$ and $\frac{1}{2}\left(\nu+\frac{1}{2}\right)$ it is evident that the inequality last written, leads to

$$
\frac{A_{2}}{\nu^{\gamma}}<\left|F_{1}(\nu)\right|<\frac{A_{1}}{\nu^{\gamma}}
$$

so that when $|\nu|$ exceeds a certain finite value in the given half-plane,

$$
\nu^{\gamma} F_{1}(\nu)=\nu^{2-2 \delta_{0}} F_{1}(\nu)
$$

is bounded.

It remains to consider the internal singular points of $f(x)$ at $x_{1}, x_{2}>\ldots$. Since these are all subject to the condition of eq $3.6 \mathrm{a}$ it is evident from the integral (eq 3.8) that they can have nothing to do with the boundary $\nu=\nu_{0}$ of the half- 
plane in which $F(\nu)$ is analytic. It may also be shown that they contribute to $F(\nu)$ terms that vanish more rapidly with increasing $\nu$ than the principal terms in eq $3.2 \mathrm{~b}$, $\mathrm{c}$, which are determined by the nature of $f(x)$ at $x=1$. The proof could be made by use of a comparison function, which is a linear combination of $f_{1}(x)$ and of $f_{2}(x)$ where

$$
f_{2}(x)=a \text { for } 1<x<x_{1},=b \text { for } x_{1}<x<\infty .
$$

The transform $F_{2}(\nu)$ is found by eq 2.1 to be

$$
F_{2}(\nu)=\frac{1}{\nu^{2}-\frac{1}{4}}\left[a+(a-b)\left(x_{1}^{2}-1\right) Q_{\nu-1 / 3}\left(x_{1}\right)\right],
$$

which is analytic in the half-plane $\frac{1}{2}=\nu_{0}<\nu_{1}$, this $\nu_{0}$ being determined by the fact that $f_{2}(x)$ is bounded. Also when $\nu \rightarrow \infty, Q_{\nu-1 / 2}(x) \rightarrow 0$, as in eq 2.18 , so $\nu^{2} F_{2}(\nu)$ is bounded (as it must be since $f_{2}(1)=a$ and $\left.\delta_{0}=0\right)$. For this example the integral representation (eq 3.4) becomes

$$
\begin{gathered}
\frac{1}{\pi i} \int_{\nu_{1}-i \infty}^{\nu_{1}+i \infty}\left[a+(a-b)\left(x_{1}^{2}-1\right) Q_{\nu-1 / 2}\left(x_{1}\right)\right] \frac{\nu P \nu_{-1 / 2}(x)}{\nu^{2}-\frac{1}{4}} d \nu= \\
a \text { for } 1 \leqslant x<x_{1},=b \text { for } x_{1}<x<\infty
\end{gathered}
$$

where $\frac{1}{2}<\nu_{1}<\infty$.

In particular

$$
\frac{1}{\pi i} \int_{\nu_{1}-i \infty}^{\nu_{1}+i \infty} \frac{\nu P_{\nu-1 / 2}(x)}{\nu^{2}-\frac{1}{4}} d \nu=1 \text { for } 1 \leqslant x<\infty
$$

where $\frac{1}{2}<\nu_{1}$

$$
\frac{1}{\pi i} \int_{\nu_{1}-i \infty}^{\nu_{1}+i \infty} \frac{\nu Q_{\nu-1 / 2}\left(x_{1}\right) P_{\nu-1 / 2}(x)}{\nu^{2}-\frac{1}{4}} d \nu=
$$$$
0 \text { for } 1 \leqslant x<x_{1},=-\frac{1}{x_{1}^{2}-1} \text { for } x_{1}<x<\infty .
$$

In general for a function $f(x)$, which becomes infinite when $x \rightarrow x_{1}-0$ in such a manner that $\int_{1}^{x_{1}-0}|f(x)| d x$ converges as in eq 3.6 a we could choose a positive constant $a$ so large that

$$
\left|\int_{1}^{x_{1}-0} f(x) Q_{\nu-1 / 2}(x) d x\right|<a \int_{1}^{x_{1}} Q_{\nu-1 / 2}(x) d x .
$$

One could show by comparison with $f_{2}(x)$ and $F_{2}(\nu)$ that the internal singular points of $f(x)$ do not affect the conditions of eq $3.2 \mathrm{a}, \mathrm{b}$, c.
The alternative assumption placed in eq $3.6 \mathrm{c}$ of a function that becomes infinite like $\log (x-1)$, when $x \rightarrow 1$ is illustrated by the third example $f_{3}(x)$, which could be used for comparison,

$$
\begin{aligned}
& f_{3}(x)=f_{3}(\cosh \beta)=\frac{1}{\Gamma(\delta)} Q_{\delta-1}(\cosh \beta)= \\
& \frac{\sqrt{\pi}}{\Gamma\left(\delta+\frac{1}{2}\right)} e^{-\delta \beta} F\left(\frac{1}{2}, \delta, \delta+\frac{1}{2} ; e^{-2 \beta}\right),
\end{aligned}
$$

which is an integral function of $\delta$ when $\beta>0$, as it then converges for any finite $\delta$. From eq 2.10 with $\nu=\delta-\frac{1}{2}$ it is evident that when $\beta \rightarrow 0(x \rightarrow 1)$,

$$
f_{3}(\beta) \sim-\frac{1}{2} \log (x-1) \sim-\log \beta .
$$

We find by - eq 2.14,

$$
F_{3}(\nu)=\frac{\psi\left(\nu+\frac{1}{2}\right)-\psi(\delta)}{\left[\nu^{2}-\left(\delta-\frac{1}{2}\right)^{2}\right] \Gamma(\delta)},
$$

which is analytic in the entire $\nu$-plane except for the pole at $\nu=\frac{1}{2}-\delta$ and those on the negative real axis at $\nu=-\left(n+\frac{1}{2}\right)$ where $n=0,1,2,3 \ldots$ The point $\nu=\delta-\frac{1}{2}$ is not a pole, since numerator and denominator of $F_{3}$ both vanish.

Hence $F_{3}(\nu)$ is analytic in the half-plane defined in eq 3.7 .

The psi-function has the asymptotic expansion

so

$$
\psi\left(\nu+\frac{1}{2}\right) \sim \log \left(\nu+\frac{1}{2}\right)+\operatorname{zero}\left(\frac{1}{\nu}\right)
$$

$$
F_{3}(\nu) \sim \frac{\log \left(\nu+\frac{1}{2}\right)}{\nu^{2} \Gamma(\delta)}+\operatorname{zero}\left(\frac{1}{\nu}\right)
$$

and $\nu \frac{1 / 2}{2} F(\nu) \sim \frac{\log \left(\nu+\frac{1}{2}\right)}{\nu^{3 / 2} \Gamma(\delta)} \rightarrow 0$ as in eq $3.2 \mathrm{~b}$. The integral representation (eq 3.4) of this function becomes, after replacing $\delta$ by $\lambda+\frac{1}{2}$ where $\lambda=\lambda_{1}+$ $i \lambda_{2}$, 
$Q_{\lambda-1 / 2}(x)=\frac{1}{\pi i} \int_{\nu_{1}-i \infty}^{\nu+i \infty} \nu\left[\frac{\psi\left(\nu+\frac{1}{2}\right)-\psi\left(\lambda+\frac{1}{2}\right)}{\nu^{2}-\lambda^{2}}\right] P_{\nu-1 / 2}$

(x) $d \nu$ for $1<x<\infty$,

where $-\lambda_{1}<\nu_{1}$ and $-\frac{1}{2}<\nu_{1}$.

A fourth example with $\delta=\frac{1}{2}$ is

$$
f_{4}(x)=\frac{\sqrt{2}}{\sqrt{x-1}} .
$$

The transform is found by use of eq 2.1 to be

$$
F_{4}(\nu)=\sqrt{2} \int_{1}^{\infty} \frac{Q_{\nu-1 / 2}}{\sqrt{x-1}}(x) d x=\frac{\pi}{\nu} .
$$

Equation 3.4 becomes

$$
\frac{\sqrt{2}}{\sqrt{x-1}}=\frac{1}{i} \int_{\nu_{1}-i_{\infty}}^{\nu_{1}+i \infty} P_{\nu-1 / 2}(x) d x \text { for } 1<x<\infty
$$

This is a special case $\alpha=0$, of

$$
\begin{aligned}
& f_{5}(x)=\frac{\sqrt{2}}{\sqrt{x-\cos \alpha}} \text { where }\left(\begin{array}{c}
1<x<\infty \\
0 \leqslant \alpha \leqslant 2 \pi
\end{array}\right) \\
& F_{5}(\nu)=\frac{\pi \cos \nu(\pi-\alpha)}{\nu \cos \nu \pi}-2 \sum_{s=0}^{\infty} \frac{\sin \left(s+\frac{1}{2}\right) \alpha}{\left(s+\frac{1}{2}\right)^{2}-\nu^{2}}= \\
& \frac{-1}{i} \int_{-i \infty}^{i \infty} \frac{\cos \lambda(\pi-\alpha)}{\left(\lambda^{2}-\nu^{2}\right) \cos \lambda \pi} d \lambda=\frac{\pi \cos \nu \pi}{\nu}+ \\
& 2 \sum_{s=0}^{\infty} \frac{\sin \nu \alpha-\sin \left(s+\frac{1}{2}\right) \alpha}{\left(s+\frac{1}{2}\right)^{2}-\nu^{2}}
\end{aligned}
$$

which is analytic in the half-plane.

$$
0<\nu_{1}<\infty \text { since } 2 \sum_{s=0}^{\infty} \frac{1}{\left(s+\frac{1}{2}\right)^{2}-\nu^{2}}=\frac{\pi \tan \nu \pi}{\nu} .
$$

The integral representation of a function $f(x)$ as in eq 3.4 and $3.5 \mathrm{a}$ are special cases $\mu=0$ of the following integral identities involving associated
Legendre functions $P_{\nu-1 / 2}^{\mu}(x)$ and $Q_{\nu-1 / 2}^{\mu}(x)$, where the constant $\mu=\mu_{1}+i \mu_{2}$ is arbitrary.

$$
\begin{aligned}
f(x)= & \frac{1}{i \pi \cos \mu \pi} \int_{\nu_{1}-i \infty}^{\nu_{1}+i \infty} \frac{\Gamma\left(\nu-\mu+\frac{1}{2}\right)}{\Gamma\left(\nu+\mu-\frac{1}{2}\right)} \nu P_{\nu-1 / 2}^{\mu}(x) d \nu \\
& \int_{1}^{\infty} f\left(x^{\prime}\right) Q_{\nu-1 / 2}^{\mu}\left(x^{\prime}\right) d x^{\prime} \text { for } 1<x<\infty,
\end{aligned}
$$

where $\frac{1}{2}-\delta<\nu_{1}<\infty$ and $-\left(\mu_{1}+\frac{1}{2}\right)<\nu_{1}<\infty$,

and in case $\delta-\frac{1}{2}>0$, for the function $f(x)$,

$$
\begin{aligned}
f(x)= & \frac{1}{i \pi \cos \mu \eta} \int_{\nu_{1}-i \infty}^{\nu_{1}+i \infty} \frac{\Gamma\left(\nu-\mu+\frac{1}{2}\right)}{\Gamma\left(\nu+\mu-\frac{1}{2}\right)} \nu Q_{\nu-1 / 2}^{\mu}(x) d \nu \\
& \int_{1}^{\infty} f\left(x^{\prime}\right) P_{\nu-1 / 2}^{\mu}\left(x^{\prime}\right) d x^{\prime}
\end{aligned}
$$

where $-\left(\delta-\frac{1}{2}\right)<\nu_{1}<\delta-\frac{1}{2}$ and $-\left(\mu_{1}+\frac{1}{2}\right)<\nu_{1}$.

In the present problem the potential has assigned values (which are axially symmetric) on the boundry of the conductor. In the more general problem these integrals would be required for $\mu=0$ or any positive integer.

Similar identities could be given in which the lower parameter $\nu$ is a constant, the upper $\mu$ being the variable of integration. I have obtained elsewhere $^{1}$ integral identities in which the development-functions are solutions of a general type of differential equation. For the range $-\infty<x<\infty$ these may be specialized to give Mellin's form of Fourier Integral, and for the range $0<x<\infty$ to give Laplace's transform.

\section{Potential With Given Values on Bound- ary of the Conductor - the function $\mathrm{S}_{\omega}$}

Let $V(\alpha, \beta)$ be a potential that vanishes at spatial infinity and has no singularities at any point in the dielectric region $D$ corresponding to the strip of figure $2, \mathrm{~b}, 0<\alpha<\omega$ and $0<\beta<\infty$.

1 The Hypergeometric and Legendre functions, NBS Mathematical Table MT15, p. 128-176 (1942). This paper is no longer available, but may be found in the larger libraries. 
At the boundary of conductor, $\alpha=0$ and $\alpha=\omega$, the potentials $V(0, \beta)$ and $V(\omega, \beta)$ are given functions of $\beta$.

Writing

$$
V(\alpha, \beta)=\sqrt{\cosh \beta-\cos (\alpha, \theta)} W(\alpha, \beta),
$$

the function of $W$ to be found is a solution of eq 1.11 without singularities in the strip of figure $2, \mathrm{~b}$, and which takes on the boundary values

$$
\left.\begin{array}{l}
W(0, \beta)=f_{1}(x)=\frac{V(0, \beta)}{\sqrt{\cosh \beta-\cos \theta}} \\
W(\omega, \beta)=f_{2}(x)=\frac{V(\omega, \beta)}{\sqrt{\cosh \beta-\cos (\omega-\theta)}}
\end{array}\right\}
$$

Since spatial infinity corresponds to $\alpha=\theta$ and $\beta=0$, eq 4.1 shows $V$ will vanish at infinity if $W(\theta, 0)$ is finite.

In determining $W$ with assigned boundary values $f_{1}(x)$ and $f_{2}(x)$, one could forget about figure 2, a and the application to potential, for it is then obvious that the width $\omega$ of the strip in figure $2, \mathrm{~b}$ may be any finite constant. Some definitions of functions that will be useful are thus obtained for $\omega=n \pi$ and $\omega=n \pi / m$, in which case the function of fundamental importance may be obtained in finite terms.

Suppose the functions $f_{1}(x)$ and $f_{2}(x)$ satisfy the conditions (3.6 a, b, c) where $\delta$ of eq $3.6 \mathrm{~b}$ is either $\delta_{1}$ or $\delta_{2}$.

If we also make the restriction

$$
\frac{1}{2}-\frac{\pi}{\omega}<\delta<\infty \quad\left(\text { where } \delta=\delta_{1} \text { or } \delta_{2}\right)
$$

this permits assigning boundary values of potential that become infinite when $\beta \rightarrow \infty$ whatever the value of $\omega$, but it only permits $f_{1}$ and $f_{2}$ to become infinite in case $0<\omega<2 \pi$.

By this condition the boundary $\nu_{1}=\nu_{0}$ of eq 3.7 of the half-plane, where eq 3.4 applies, lies to the left of $\nu_{1}=\pi / \omega$. Hence the required $W$-function is by eq 3.4

$$
\begin{array}{r}
W(\alpha \beta)=\frac{1}{\pi i} \int_{\pi / \omega-0-i \infty}^{\pi / \omega-0+i \infty} \nu P_{\nu-1 / 2}(x) d \nu\left\{\frac{\sin \nu(\omega-\alpha)}{\sin \nu \omega} \int_{1}^{\infty} f_{1}\left(x^{\prime}\right) Q_{\nu-1 / 2}\left(x^{\prime}\right) d x^{\prime}+\frac{\sin \nu \alpha}{\sin \nu \omega} \int_{1}^{\infty} f_{2}\left(x^{\prime}\right) Q_{\nu-1 / 2}\left(x^{\prime}\right) d x^{\prime}\right\}(4.4) \\
W(\alpha, \beta)=\int_{1}^{\infty} d x^{\prime} \frac{1}{\pi i} \int_{\pi / \omega-0-i \infty}^{\pi / \omega-0+i \infty} \nu P_{\nu-1 / 2}(x) Q_{\nu-1 / 2}\left(x^{\prime}\right)\left[\frac{\sin \nu(\omega-\alpha)}{\sin \nu \omega} f_{1}\left(x^{\prime}\right)+\frac{\sin \nu \alpha}{\sin \nu \omega} f_{2}\left(x^{\prime}\right)\right] d \nu=D_{\alpha}\left\{\int_{1}^{\infty} f_{1}\left(x^{\prime}\right) \frac{d x^{\prime}}{\pi i}\right. \\
\left.\int_{\nu 1-i \infty}^{\nu_{1}+i \infty} \frac{\cos \nu(\omega-\alpha)}{\sin \nu \omega} P_{\nu-1 / 2}(x) Q_{\nu-1 / 2}\left(x^{\prime}\right) d \nu-\int_{1}^{\infty} f_{2}\left(x^{\prime}\right) \frac{d x^{\prime}}{\pi i} \int_{\nu 1-i \infty}^{\nu_{1}+i \infty} \frac{\cos \nu \alpha}{\sin \nu \omega} P_{\nu-1 / 2}(x) Q_{\nu-1 / 2}\left(x^{\prime}\right) d \nu\right\} .
\end{array}
$$

where $0<\nu_{1}<\pi / \omega$.

This may be written

$$
W(\alpha, \beta)=\frac{1}{2} D_{\alpha} \int_{1}^{\infty}\left\{-f_{1}\left(x^{\prime}\right) S_{\omega}\left(\alpha, \beta, \beta^{\prime}\right)+f_{2}\left(x^{\prime}\right) S_{\omega}\left(\omega-\alpha, \beta, \beta^{\prime}\right)\right\} d x^{\prime} .
$$

In this equation the function $S_{\omega}\left(\alpha, \beta, \beta^{\prime}\right)$ is by definition an even periodic function of $\alpha$ with

$$
S_{\omega}(\alpha, \beta, \beta) \equiv \frac{1}{i} \int_{\nu_{1}-i \infty}^{\nu_{1}+i \infty} \frac{\cos \nu(\omega-|\alpha|)}{\sin \nu \omega} \tan \nu \pi P_{\nu-1 / 2}(\cosh \beta) P_{\nu-1 / 2}\left(\cosh \beta^{\prime}\right) d \nu,
$$

where the path ${ }_{\nu 1}=$ constant is in the narrower of the two strips,

$$
\left.\begin{array}{l}
-\frac{1}{2}<\nu_{1}<\frac{1}{2}(\text { so } \tan \pi \neq \infty) \\
-\pi / \omega<\nu_{1}<\pi / \omega(\text { so } 1 / \sin \nu \omega \neq \infty)
\end{array}\right\}
$$

(When $0<\omega<2 \pi$ ), the first is the narrower). By the method used in getting eq 2.30 from eq 2.29 it is found that eq 4.6 is equivalent to

$$
S_{\omega}\left(\alpha, \beta, \beta^{\prime}\right)=\frac{2}{\omega} Q_{-1 / 2}(\cosh \beta) P_{-1 / 2}\left(\cosh \beta^{\prime}\right)-\frac{2}{\pi i} \int_{\nu_{1}-i \infty}^{\nu_{1}+i \infty} \frac{\cos \nu(\omega-|\alpha|)}{\sin \nu \omega} Q_{\nu-1 / 2}(\cosh \beta) P_{\nu-1 / 2}\left(\cosh \beta^{\prime}\right) d \nu,
$$

where $0<\nu_{1}<\pi / \omega$.

The same is true with $\beta$ and $\beta^{\prime}$ interchanged. By

$$
S_{\omega}\left(\alpha, \beta, \beta^{\prime}\right)=\frac{4}{\omega} \sum_{n=0}^{\infty} \epsilon_{n} Q_{n \pi / \omega-1 / 2}\left(\cosh \beta^{\prime}\right) P_{n \pi / \omega-1 / 2}(\cosh \beta) \cos \frac{n \pi \alpha}{\omega},
$$


When $0 \leqslant \beta \leqslant \beta^{\prime}$; otherwise these are to be interchanged.

The form (4.8) shows immediately the equivalence of eq 4.4 and 4.5. The singular points of $S_{\omega}$ are at $\alpha= \pm 2 n \pi / \omega, \beta=\beta^{\prime}$, where $n=0,1,2$, .... Hence if $\pi / \omega$ is irrational the functions $S_{\omega}$ and $S_{\pi}$ have only one singular point in common (at $\alpha=0, \beta=\beta^{\prime}$ ).

The period interval $-\omega<\alpha<\omega$ was taken for the definition of $S_{\omega}$ in eq 4.6 in order that

$$
\frac{\cos \nu(\omega-|\alpha|)}{\sin \nu \omega} \rightarrow 0 \text { when } \nu_{2} \rightarrow \pm \infty .
$$

For some purposes it is more convenient to consider $\alpha$ in the interval $0<\alpha<2 \omega$, so that the singular points are on the boundary of the interval. Any expression that represents $S \omega$ for the interval $0<\alpha \leqslant \omega$ is sufficient to determine $S_{\omega}$ for all values of $\alpha$. The periodicity combined with the fact that $S_{\omega}$ is an even function of $\alpha$ gives

$$
S_{\omega}\left(2 \omega-\alpha, \beta, \beta^{\prime}\right)=S_{\omega}\left(\alpha, \beta, \beta^{\prime}\right)
$$

and

$$
\begin{aligned}
D_{\alpha} S_{\omega}\left(\alpha, \beta, \beta^{\prime}\right) & =0 \text {, when } \alpha= \pm \omega \\
& =0 \text {, when } \alpha=0 \text {, or } 2 \omega \text { if } \beta \neq \beta^{\prime} \\
& =-\infty \text { when } \alpha \rightarrow+0 \text { if } \beta=\beta^{\prime} \\
& =+\infty \text { when } \alpha \rightarrow 2 \omega-0 \text { if } \beta=\beta^{\prime} .
\end{aligned}
$$

The functions $S_{\omega}\left(\alpha, \beta, \beta^{\prime}\right)$ and $S_{\pi}\left(\alpha, \beta, \beta^{\prime}\right)$ each go to $+\infty$ when $\alpha \rightarrow 0$ and $\beta \rightarrow \beta^{\prime}$, but their difference remains finite.

When $\alpha \rightarrow 0$ and $\beta \rightarrow \beta^{\prime}>0$, both functions become logarithmically infinite (since the singularity is a line-charge).

$$
\begin{array}{ll}
S_{\omega}\left(\alpha, \beta, \beta^{\prime}\right) \sim S_{\pi}\left(\alpha, \beta, \beta^{\prime}\right) \sim \frac{-1}{\pi \sinh \beta} & S_{\omega}\left(\alpha+\frac{2 \omega t}{m}\right)=\frac{4}{\omega} \sum_{n=0}^{\infty} \epsilon_{n} F\left(\frac{n \pi}{\omega}\right) \\
\log \sqrt{\alpha^{2}+\left(\beta-\beta^{\prime}\right)^{2}}, & \text { (4.11a) }
\end{array}
$$

plus terms that remain finite. In case $\beta^{\prime}=0$ and $\alpha \rightarrow 0, \beta \rightarrow 0$, the singularity is a point-charge and, as in eq 2.27 , both became finite.

$S_{\omega}(\alpha, \beta, 0) \sim S_{\pi}(\beta, \beta, 0) \sim \frac{2}{\sqrt{\alpha^{2}+\beta^{2}}}+$ finite terms.

Subtracting eq 2.29 from eq 4.6 gives (taking the path up the imaginary axis of $\nu$ )

$$
\begin{aligned}
& S_{\omega}\left(\alpha, \beta, \beta^{\prime}\right)-S_{\pi}\left(\alpha, \beta, \beta^{\prime}\right)= \\
& \frac{1}{i} \int_{i \infty}^{i \infty}\left[\cos \nu(\omega-|\alpha|) \frac{\sin \nu \pi}{\sin \nu \omega}-\cos \nu(\pi-|\alpha|)\right] \\
& \frac{P_{\nu-1 / 2}(\cosh \beta) P_{\nu-1 / 2}\left(\cosh \beta^{\prime}\right)}{\cos \nu \pi} d \nu,
\end{aligned}
$$

where $|\alpha|<2 \pi$ and $|\alpha|<2 \omega$.

For $\alpha=0$, this becomes

$$
\begin{aligned}
& S_{\omega}\left(0, \beta, \beta^{\prime}\right)-S_{\pi}\left(0, \beta, \beta^{\prime}\right)= \\
& \frac{1}{i} \int_{i \infty}^{i \infty}\left[\frac{\tan \nu \pi}{\tan \nu \omega}-1\right] P_{\nu-1 / 2}(x) P_{\nu-1 / 2}\left(x^{\prime}\right) d \nu .
\end{aligned}
$$

Since the bracket vanishes when $\nu \rightarrow \pm \infty$, this series converges, as shown by eq 2.21 , if $\beta$ and $\beta^{\prime}$ are not both zero. It will be shown in a following section that eq $4.11 \mathrm{~b}$ is true.

The Fourier series (eq 4.9) is of the form

$$
\frac{4}{\omega} \sum_{n=0}^{\infty} A_{\omega, \mathrm{n}} \cos (n \pi \alpha / \omega)
$$

where the coefficients $A_{\omega, n}$ depend upon $\omega$ and $n$ in the particular form as a function of $n \pi / \omega$ or (suppressing $\beta$ and $\beta^{\prime}$ )

$$
S_{\omega}(\alpha)=\frac{4}{\omega} \sum_{n=0}^{\infty} \epsilon_{n} F\left(\frac{n \pi}{\omega}\right) \cos \left(\frac{n \pi \alpha}{\omega}\right) .
$$

Let $m$ be a positive integer, and replace $\alpha$ by $\alpha+\frac{2 \omega}{m} t$,

$$
S_{\omega}\left(\alpha+\frac{2 \omega t}{m}\right)=\frac{4}{\omega} \sum_{n=0}^{\infty} \epsilon_{n} F\left(\frac{n \pi}{\omega}\right) \cos \frac{n \pi}{\omega}\left(\alpha+\frac{2 \omega t}{m}\right) .
$$


Hence

$$
\begin{aligned}
\sum_{t=0}^{m-1} S_{\omega}\left(\alpha+\frac{2 \omega t}{m}\right) & =\frac{4}{\omega / m} \sum_{k=0}^{\infty} \epsilon_{k} F\left(\frac{k \pi}{\omega / m}\right) \cos \left(\frac{k \pi \alpha}{\omega / m}\right) \\
& =S_{\omega / m}(\alpha) .
\end{aligned}
$$

That is,

$$
S_{\omega / m}\left(\alpha, \beta, \beta^{\prime}\right)=\sum_{t=0}^{m-1} S_{\omega}\left(\alpha+\frac{2 \omega t}{m}, \beta, \beta^{\prime}\right) .
$$

This may be written in the following forms in which the argument of every $S$-function lies in its first, positive, half-period interval.

$$
\text { For } 0<\alpha \leqslant \frac{\omega}{2 m-1} \text { and } m>1 \text {, }
$$

$S_{\omega /(2 m-1)}\left(\alpha, \beta, \beta^{\prime}\right)=S_{\omega}\left(\alpha, \beta, \beta^{\prime}\right)+$

$\sum_{t=1}^{m-1}\left[S_{\omega}\left(\frac{2 t \omega}{2 m-1}+\alpha, \beta, \beta^{\prime}\right)+S_{\omega}\left(\frac{2 t \omega}{2 m-1}-\alpha, \beta, \beta^{\prime}\right)\right]$

and for $0<\alpha \leqslant \frac{\omega}{2 m}$ where $m \geqslant 1$,

$S_{\omega / 2 m}\left(\alpha, \beta, \beta^{\prime}\right)=S_{\omega}\left(\alpha, \beta, \beta^{\prime}\right)+S_{\omega}\left(\omega-\alpha, \beta, \beta^{\prime}\right)+$
$\sum_{t=1}^{\varepsilon-1}\left[S_{\omega}\left(\frac{t \omega}{m}+\alpha, \beta, \beta^{\prime}\right)+S_{\omega}\left(\frac{t \omega}{m}-\alpha, \beta, \beta^{\prime}\right)\right]$,

where the sum is absent in the case $m=1$.

Taking $\omega=\pi$, gives (by eq 2.32) $S_{\pi / m}\left(\alpha, \beta, \beta^{\prime}\right.$ ) in terms of a finite number of complete elliptic integrals.

In particular for $\beta^{\prime}=0$, this gives, by use of eq 2.31 ,

$$
S_{\pi / m}(\alpha, \beta, 0)=\sqrt{2} \sum_{t=0}^{\infty} \frac{1}{\sqrt{\cosh \beta-\cos (\alpha+2 \pi t / m)}},
$$

where the radical is always positive.

\section{Grounded Conductor Under the Influ- ence of a Circular Line-Charge,Green's Function}

A total charge $M^{\prime}$ in the shape of a circular linecharge coaxial with the $x$-axis has for its trace a point $P^{\prime}\left(x^{\prime}, \rho^{\prime}\right)$ in the half-plane of figure 2 , a or $P^{\prime}\left(\alpha^{\prime}, \beta^{\prime}\right)$ in the $\zeta$-strip. Consider $P^{\prime}$ in the dielectric region $(D) \quad\left(0<\alpha^{\prime}<\omega\right)$. The potential of this line-charge, together with that of the charges it induces on the conductor (at zero potential), is given at any point $(\alpha, \beta)$ in $(D)$ by

$$
V_{\omega}\left(\alpha, \beta ; \alpha^{\prime}, \beta^{\prime}\right)=\frac{M^{\prime}}{2 c} \sqrt{[\cosh \beta-\cos (\alpha-\theta)]\left[\cosh \beta^{\prime}-\cos \left(\alpha^{\prime}-\theta\right)\right]} W_{\omega}\left(\alpha, \beta ; \alpha^{\prime}, \beta^{\prime}\right),
$$

where

$$
W_{\omega}\left(\alpha, \beta, \beta^{\prime}\right)=S_{\omega}\left(\alpha-\alpha^{\prime}, \beta, \beta^{\prime}\right)-S_{\omega}\left(\alpha+\alpha^{\prime}, \beta, \beta^{\prime}\right) .
$$

Proof: The second Ifunction with positive argument, $\alpha+\alpha^{\prime}<2 \omega$, has no singularity in this periodinterval. The first has only one (at $\alpha=\alpha^{\prime}, \beta=\beta^{\prime}$ ), and by eq $4.10 \mathrm{a}$, together with eq 2.24 , it follows that $S_{\omega}$ at this point differs from $S_{\pi}$ there by finite terms.

The potential $V_{\omega}$ vanishes at spatial infinity $(\alpha=\theta, \beta=0)$ since the radical in eq 5.1 then vanishes.

Also $W_{\omega}$ vanishes at the conducting boundary, $\alpha=0$, because $S_{\omega}$ is an even function of its first argument. At the remaining boundary $W_{\omega}$ vanishes since $S_{\omega}$ is also periodic in its first argument with period $2 \omega$.

Hence this $W$-function is the Green's function for eq 1.11 whatever the value of $\omega$. The potential $V_{\omega}$ is the Green's function for the potential equation if $0<\omega<2 \pi$.
Using the series (4.9) in eq 5.2, gives for $0 \leqslant \alpha \leqslant \omega$,

$W_{\omega}\left(\alpha, \beta ; \alpha^{\prime}, \beta^{\prime}\right)=\frac{8}{\omega} \sum_{n=1}^{\infty} Q_{\frac{n \pi}{\omega}-\frac{1}{2}}\left(\cosh \beta^{\prime}\right) P_{\frac{n \pi}{\omega}-\frac{1}{2}}(\cosh \beta)$

$\sin \left(\frac{n \pi \alpha}{\omega}\right) \sin \left(\frac{n \pi \alpha^{\prime}}{\omega}\right)$

when $0 \leqslant \beta \leqslant \beta^{\prime}$, otherwise $\beta$ and $\beta^{\prime}$ are to be interchanged.

If $r=\sqrt{x^{2}+\rho^{2}}$, eq 1.5 leads to

$$
r \sqrt{\cosh \beta-\cos (\alpha-\theta)}=c \sqrt{2} \sqrt{\frac{\cos (\alpha-\theta)}{1-c^{2} / r^{2}}} .
$$

The total charge $M$ induced in the conductor is given by

$$
M+M^{\prime}=\lim _{r \rightarrow \infty}\left(r V_{\omega}\right)
$$

so by eq 5.1 we get 


$$
M=-M^{\prime} \sqrt{\frac{\cosh \beta^{\prime}-\cos \left(\alpha^{\prime}-\theta\right)}{2}}\left[\frac{\sqrt{2}}{\sqrt{\cosh \beta^{\prime}-\cos \left(\alpha^{\prime}-\theta\right)}}+S_{\omega}\left(\theta+\alpha^{\prime}, 0, \beta^{\prime}\right)-S_{\omega}\left(\theta-\alpha^{\prime}, 0, \beta^{\prime}\right)\right] .
$$

The surface density of charge on the boundary of the conductor is a function of $\cosh \beta$ defined by $4 \pi \sigma(\cosh \beta)=\partial V / \partial n$, where $d n$ is the linear element normal to the conductor, pointing toward it. By eq $1.17, d n=\frac{-c d \alpha}{\cosh \beta-\cos \theta}$ at the $\operatorname{arc} \alpha=0$ and $=\frac{+c d \alpha}{\cosh \beta-\cos (\omega-\theta)}$ at the arc $\alpha=\omega$.

On the first arc, $\alpha=0$, the density is given by

$$
2 \pi \sigma_{1}(\cosh \beta)=\frac{M^{\prime}}{c^{2} \sqrt{2}} \sqrt{\frac{\cosh \beta^{\prime}-\cos \left(\alpha^{\prime}-\theta\right)}{2}}
$$$$
(\cosh \beta-\cos \theta)^{3 / 2} D_{\alpha^{\prime}} S_{\omega}\left(\alpha^{\prime}, \beta, \beta^{\prime}\right) .
$$

On the second are,

$$
2 \pi \sigma_{2}(\cosh \beta)=\frac{-M^{\prime}}{c^{2} \sqrt{2}} \sqrt{\frac{\cosh \beta^{\prime}-\cos \left(\alpha^{\prime}-\theta\right)}{2}}
$$

$(\cosh \beta-\cos (\omega-\theta))^{3 / 2} D_{\alpha^{\prime}} S_{\omega}\left(\omega-\alpha^{\prime}, \beta, \beta^{\prime}\right)$.

From here on we consider $\beta^{\prime}=0$ and write

$$
S_{\omega}(\alpha, \beta) \text { for previous } S_{\omega}(\alpha, \beta, 0) \text {. }
$$

When the radius $\rho^{\prime}$ of the line-charge shrinks to zero, there is then a point charge $M^{\prime}$ at $P^{\prime}\left(\alpha^{\prime}, 0\right)$ on the $x$-axis. The potential becomes

$$
\begin{aligned}
& V_{\omega}\left(\alpha, \beta, \alpha^{\prime}, 0\right)=\frac{M^{\prime}\left|\sin \left(\frac{\alpha^{\prime}-\theta}{2}\right)\right|}{c \sqrt{2}} \\
& {[\cosh \beta-\cos (\alpha-\theta)]^{1 / 2} W_{\omega}\left(\alpha, \beta ; \alpha^{\prime}, 0\right),}
\end{aligned}
$$

where

$$
W_{\omega}\left(\alpha, \beta ; \alpha^{\prime}, 0\right)=S_{\omega}\left(\alpha-\alpha^{\prime}, \beta\right)-S_{\omega}\left(\alpha+\alpha^{\prime}, \beta\right) .
$$

The induced charge (eq 5.5) becomes

$$
\begin{gathered}
M=-M^{\prime}\left|\sin \frac{\alpha^{\prime}-\theta}{2}\right|\left[S_{\omega}\left(\alpha^{\prime}+\theta, 0\right)-\right. \\
\left.S \omega\left(\alpha^{\prime}-\theta, 0\right)+\frac{1}{\left|\sin \frac{\alpha^{\prime}-\theta}{2}\right|}\right] .
\end{gathered}
$$

The surface densities become

$$
2 \pi \sigma_{1}(\cosh \beta)=\frac{M^{\prime}\left|\sin \left(\frac{\alpha^{\prime}-\theta}{2}\right)\right|}{c^{2} \sqrt{2}}
$$$$
[\cosh \beta-\cos \theta]^{3 / 2} D_{\alpha^{\prime}} S_{\omega}\left(\alpha^{\prime}, \beta\right) ;
$$

$$
2 \pi \sigma_{2}(\cosh \beta)=\frac{-M^{\prime}\left|\sin \left(\frac{\alpha^{\prime}-\theta}{2}\right)\right|}{c^{2} \sqrt{2}}
$$

$[\cosh \beta-\cos (\omega-\theta)]^{3 / 2} D_{\alpha^{\prime}} S_{\omega}\left(\omega-\alpha^{\prime}, \beta\right)$.

For comparison with this, the logarithmic potential $v_{\omega}\left(\alpha, \beta ; \alpha^{\prime}, \beta^{\prime}\right)$, which is a solution of $\left(D_{\alpha}^{2}+D_{\beta}^{2}\right) v=0$, analogous to eq 5.1 , corresponds to two endless line-charges each of charge $m^{\prime} / 2$ per unit length, perpendicular to the plane of figure 1 .

The conductor is now bounded by two endless circular cylinders with trace $\alpha=0$ and $\alpha=\omega$. The two parallel line-charges are at $P^{\prime}\left(x^{\prime}, \rho^{\prime}\right.$ and $P^{\prime \prime}\left(x^{\prime},-\rho^{\prime}\right)$, that is, $P^{\prime}\left(\alpha^{\prime}, \beta^{\prime}\right)$ and $P^{\prime \prime}\left(\alpha^{\prime},-\beta^{\prime}\right)$. The potential is

$$
\begin{aligned}
& v_{\omega}\left(\alpha, \beta ; \alpha^{\prime}, \beta^{\prime}\right)= \\
& \frac{m^{\prime}}{2} \log \left[\frac{\cosh \pi\left(\beta-\beta^{\prime}\right) / \omega-\cos \pi\left(\alpha+\alpha^{\prime}\right) / \omega}{\cosh \pi\left(\beta-\beta^{\prime}\right) / \omega-\cos \pi\left(\alpha-\alpha^{\prime}\right) / \omega}\right]+ \\
& \frac{m^{\prime}}{2} \log \left[\frac{\cosh \pi\left(\beta+\beta^{\prime}\right) / \omega-\cos \pi\left(\alpha+\alpha^{\prime}\right) / \omega}{\cosh \pi\left(\beta+\beta^{\prime}\right) / \omega-\cos \pi\left(\alpha-\alpha^{\prime}\right) / \omega}\right] .
\end{aligned}
$$

When the two line-charges come together to make one of strength $m^{\prime}$ with trace at $P^{\prime}\left(\alpha^{\prime}, 0\right)$ on the $x$-axis, this becomes

$$
\begin{aligned}
& v_{\omega}\left(\alpha, \beta ; \alpha^{\prime}, 0\right)= \\
& m^{\prime} \log \left[\frac{\cosh \pi \beta / \omega-\cos \pi\left(\alpha+\alpha^{\prime}\right) / \omega}{\cosh \pi \beta / \omega-\cos \pi\left(\alpha-\alpha^{\prime}\right) / \omega}\right] .
\end{aligned}
$$

The charge induced on the cylinder is $-m^{\prime}$ per unit length. It is distributed with surface densities, per unit length, perpendicular to the plane of figure 1 , on arc $\alpha=0$,

$2 \pi \sigma_{1}(\cosh \beta)=\frac{-m^{\prime} \pi}{c \omega} \sin \frac{\pi \alpha^{\prime}}{\omega}\left[\frac{\cosh \beta-\cos \theta}{\cosh \pi \beta / \omega-\cos \pi \alpha^{\prime} / \omega}\right]$, and on $\operatorname{arc} \alpha=\omega$,

$$
2 \pi \sigma_{2}(\cosh \beta)=\frac{-m^{\prime} \pi}{c \omega} \sin \frac{\pi \alpha^{\prime}}{\omega}\left[\frac{\cosh \beta-\cos (\omega-\theta}{\cosh \pi \beta / \omega+\cos \pi \alpha^{\prime} / \omega}\right] .
$$


VI. Potential of the Charged Conductor Alone

The formulation of this potential will be simpler if we first evaluate a limiting case of the $S$-function. When $\beta^{\prime}=0$, this is given by the second series (eq 4.9) for all values of $\alpha$ and all positive values of $\beta$,

$$
S_{\omega}(\alpha, \beta)=\frac{4}{\omega} \sum_{n=0}^{\infty} \epsilon_{n} Q_{\frac{n \pi}{\omega}-\frac{1}{2}}(\cosh \beta) \cos \frac{n \pi \alpha}{\omega} . \quad \sum_{n=0}^{\infty} 2 \epsilon_{n} e^{-n \pi \beta^{\prime} / \omega} \operatorname{ccs} n \pi \alpha / \omega=\frac{\sinh \pi \beta^{\prime} / \omega}{\cosh \left(\pi \beta^{\prime} \omega\right)-\cos \pi \alpha / \omega},
$$

Replacing the $Q$-functions by their equivalent integral expressions given in eq 2.23 leads to $S_{\omega}(\alpha, \beta)=$

$\frac{\sqrt{2}}{\omega} \int_{\beta}^{\infty} \frac{d \beta^{\prime}}{\sqrt{\cosh \beta^{\prime}-\cosh \beta}} \sum_{n=0}^{\infty} 2 \epsilon_{n} e^{-n \pi \beta^{\prime} \omega} \cos n \pi \alpha / \omega$.

Since

this becomes

$$
S_{\omega}(\alpha, \beta)=\frac{\sqrt{2}}{\omega} \int_{\beta}^{\infty} \frac{\sinh \left(\pi \beta^{\prime} / \omega\right) d \beta^{\prime}}{\left.\cosh \frac{\pi \beta^{\prime}}{\omega}-\cos \frac{\pi \alpha}{\omega}\right] \sqrt{\cosh \beta^{\prime}-\cosh \beta}}
$$

or

$$
S_{\omega}(\alpha, \beta)=\frac{\sqrt{2}}{\pi} \int_{\pi \beta / \omega}^{\infty} \frac{\sinh x d x}{\left[\cosh x-\cos \frac{\pi \alpha}{\omega}\right] \sqrt{\cosh \frac{\omega x}{\pi}-\cosh \beta}},
$$

or

$$
S_{\omega}(\alpha, \beta)=\frac{2}{\pi} \int_{\frac{\pi \beta}{2 \omega}}^{\infty} \frac{\sinh x \cosh x d x}{\left[\cosh ^{2} x-\cos ^{2} \frac{\pi \alpha}{2 \omega}\right] \sqrt{\sinh ^{2} \frac{\omega x}{\pi}-\sinh ^{2} \frac{\beta}{2}}} .
$$

These integrals converge except at singular points, $\alpha=2 n \omega, \beta=0$.

To put in evidence the nature of the singularity of $S_{\omega}$, we may now define a function $G_{\omega}(\alpha, \beta)$ that is finite for all real values of $\alpha, \beta$, and $\omega$, and is an even periodic function of $\alpha$ with period $2 \omega$.

$$
\begin{gathered}
G_{\omega}(\alpha, \beta) \equiv S_{\omega}(\alpha, \beta)-\frac{\pi}{\omega \sqrt{\cosh ^{2} \frac{\pi \beta}{2 \omega}-\cos ^{2} \frac{\pi \alpha}{2 \omega}}}= \\
S_{\omega}(\alpha, \beta)-\frac{\pi}{\omega} S_{\pi}\left(\frac{\pi \alpha}{\omega}, \frac{\pi \beta}{\omega}\right) .
\end{gathered}
$$

To show this replace $\omega$ by $\pi$ in eq $6.1 \mathrm{c}$ and then replace $\alpha$ and $\beta$ by $\pi \alpha / \omega$ and $\pi \beta / \omega$. This gives, after multiplying by $\pi / \omega$,

$$
\begin{aligned}
\frac{\pi}{\omega} S_{\omega}\left(\frac{\pi \alpha}{\omega}, \frac{\pi \beta}{\omega}\right)= & \frac{\pi}{\omega \sqrt{\cosh ^{2} \frac{\pi \beta}{2 \omega}-\cos ^{2} \frac{\pi \alpha}{2 \omega}}}=\frac{4}{\omega} \sum_{n=0}^{\infty} \epsilon_{n} \cos \left(\frac{n \pi \alpha}{\omega}\right) Q_{n-1 / 2}\left(\cosh \frac{\pi \beta}{\omega}\right)= \\
& \frac{2}{\pi} \int_{\frac{\pi \beta}{2 \omega}}^{\infty} \frac{\sinh x \cosh x d x}{\left[\cosh ^{2} x-\cos ^{2} \frac{\pi \alpha}{2 \omega}\right] \sqrt{\sinh ^{2} x-\sinh ^{2} \frac{\pi \beta}{2 \omega}}} \cdot
\end{aligned}
$$

Substracting this from eq. 6.1 gives

$$
G_{\omega}(\alpha, \beta)=\frac{2}{\pi} \int_{\frac{\pi \beta}{2 \omega}}^{\infty}\left[\cosh \frac{\sinh x \cosh x}{\cos ^{2} \frac{\pi \alpha}{2 \omega}}\right]\left[\frac{1}{\sqrt{\sinh ^{2} \frac{\omega x}{\pi}-\sinh ^{2} \beta / 2}}-\frac{\pi}{\omega \sqrt{\sinh ^{2} x-\sinh ^{2} \frac{\pi \beta}{2 \omega}}}\right] d x,
$$

which becomes when $\beta=0$

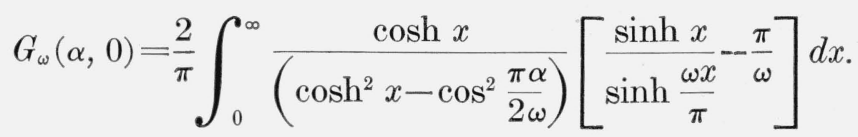


This converges throughout the period interval, boundaries included, $0 \leqslant \alpha \leqslant 2 \omega$. This is evident from the fact that when $\cos ^{2}(\pi \alpha / 2 \omega)=1$, the value of the integrand at the lower limit, $x=0$, is the finite quantity $\frac{1}{6}\left(\frac{\pi}{\omega}-\frac{\omega}{\pi}\right)$.

Using the series in eq 6.3 gives

$$
\begin{gathered}
G_{\omega}(\alpha, \beta)=\frac{4}{\omega} \sum_{n=0}^{\infty} \epsilon_{n} \cos \frac{n \pi \alpha}{\omega} \\
{\left[Q_{n \pi / \omega-1 / 2}(\cosh \beta)-Q_{n-1 / 2}\left(\cosh \frac{\pi \beta}{\omega}\right)\right] .}
\end{gathered}
$$

When $\beta \rightarrow 0$, we find from 2.10 ,

$$
\begin{aligned}
& \lim _{\beta \rightarrow 0}\left[Q_{n \pi / \omega-1 / 2}(\cosh \beta)-Q_{n-1 / 2}\left(\cosh \frac{\pi \beta}{\omega}\right)\right]= \\
& \psi\left(n+\frac{1}{2}\right)-\psi\left(\frac{n \pi}{\omega}+\frac{1}{2}\right)+\log \frac{\pi}{\omega},
\end{aligned}
$$

so

$$
\begin{aligned}
G_{\omega}(\alpha, 0)= & \frac{4}{\omega} \sum_{n=0}^{\infty} \epsilon_{n} \cos \frac{n \pi \alpha}{\omega} \\
& {\left[\psi\left(n+\frac{1}{2}\right)-\psi\left(\frac{n \pi}{\psi}+\frac{1}{2}\right)+\log \frac{\pi}{\psi}\right] . }
\end{aligned}
$$

When $n$ is large this bracket is [ ] $\sim \frac{1-\omega^{2} / \pi^{2}}{24 n^{2}}$, which shows that the series is absolutely convergent for any real $\alpha$.

Now consider the potential (5.8a), which is induced by the point-charge $M^{\prime}$. The charge, $M$. induced on the conductor will vanish if the pointcharge of constant 'strength, $M^{\prime}$, is moved to spatial infinity $\left(\alpha^{\prime} \rightarrow \theta\right)$.

However, if the strength $M^{\prime}$ of the point-charge is allowed to increase as it recedes, and in such a manner that

$$
\lim _{\alpha^{\prime} \rightarrow \theta} \frac{M^{\prime}}{c}\left|\sin \frac{\alpha^{\prime}-\theta}{2}\right|=-V^{0}=\lim _{x^{\prime} \rightarrow \infty} \frac{M^{\prime}}{\sqrt{\left(x^{\prime}\right)^{2}+c^{2}}}
$$

then the induced charge, $M$, on the conductor will approach a finite limit, which by eq $5.8 \mathrm{c}$ is

$$
M=c V^{0}\left\{S_{\omega}(2 \theta, 0)+\lim _{\alpha^{\prime} \rightarrow \theta}\left[\frac{1}{\left|\sin \frac{\alpha^{\prime}-\theta}{2}\right|}-S_{\omega}\left(\alpha^{\prime}-\theta, 0\right)\right]\right\} \text {. }
$$

This limit exists and may be put in terms of the function $G_{\omega}$, for

$$
\lim _{x \rightarrow 0}\left[\frac{a}{\sin a x}-\frac{1}{\sin x}\right]=\lim _{x=0}\left(\frac{\alpha^{2}-1}{6}\right) x=0 .
$$

Hence taking $x=\left(\alpha^{\prime}-\theta\right) / 2$ and $a=\pi / \omega$, it is evident that

$\lim _{\alpha^{\prime} \rightarrow \theta}\left[\frac{1}{\left|\sin \frac{\alpha^{\prime}-\theta}{2}\right|}-S_{\omega}\left(\alpha^{\prime}-\theta, 0\right)\right]=-G_{\omega}(0,0)$,

which is finite, so that

$$
\lim _{\alpha \rightarrow \theta}\left|\sin \left(\frac{\alpha-\theta}{2}\right)\right| S_{\omega}(\alpha-\theta, 0)=1 .
$$

We thus find, without integration, the following expression for the total charge $M$ on the conductor existing alone (and therefore at constant potential).

$$
M=c V^{0}\left[S_{\omega}(2 \theta, 0)-G_{\omega}(0,0)\right],
$$

or by eq 6.2 ,

$$
M=c V^{0}\left[\frac{\pi}{\omega \sin (\pi \theta / \omega)}+G_{\omega}(2 \theta, 0)-G_{\omega}(0,0)\right] .
$$

The potential (5.8 a) belonging to this charge becomes

$$
\begin{aligned}
V(\alpha, \beta)= & -V^{0} \sqrt{\frac{\cosh \beta-\cos (\alpha-\theta)}{2}}\left[S_{\omega}(\alpha-\theta, \beta)-\right. \\
& \left.S_{\omega}(\alpha+\theta, \beta)\right] .
\end{aligned}
$$

This vanishes at the conducting boundary $\alpha=0$ because $S_{\omega}$ is an even function of $\alpha$. It vanishes at the other boundary, $\alpha=\omega$, because $S_{\omega}$ is periodic in $\alpha$ with period $2 \omega$.

At spatial infinity $\alpha=\theta, \beta=0$, it takes the form $\infty .0$, but by eq $6.8 \mathrm{~b}$ this is just $-V^{0}$. Hence by adding the constant $V^{0}$ we find the Newtonian potential of the charged conductor alone, in the form

$$
\begin{aligned}
V(\alpha, \beta)= & V^{0}\left\{1-\sqrt{\frac{\cosh \beta-\cos (\alpha-\theta)}{2}}\left[S_{\omega}(\alpha-\theta, \beta)-\right.\right. \\
& \left.\left.S_{\omega}(\alpha+\theta, \beta)\right]\right\} .
\end{aligned}
$$

This may be written, by use of eq 4.9, since $\beta^{\prime}=0$ by eq 5.7 ,

$$
V(\alpha, \beta)=V^{0}-V^{0} \sqrt{\frac{\cosh \beta-\cos (\alpha-\theta)}{2}} \cdot \frac{8}{\pi} \sum_{n-1}^{\infty} \frac{Q_{\frac{n \pi}{\omega}-\frac{1}{2}}}{2}(\cosh \beta) \sin \frac{n \pi \theta}{\omega} \sin \frac{n \pi \alpha}{\omega} .
$$


By use of eq 4.8 with $\beta^{\prime}=0$, this may be written as an integral. The occurance of the absolute value $|\alpha-\theta|$ in the integral presents two cases (see fig. 2, a).

\section{Case $1(0 \leqslant \alpha \leqslant \theta)$}

$V(\alpha, \beta)=V^{0}$

$$
\begin{aligned}
& V^{0} \sqrt{\frac{\cosh \beta-\cos (\alpha-\theta)}{2}} . \\
& \frac{4}{\pi i} \int_{\nu_{1}-i \infty}^{\nu_{1}+i \infty} \frac{\sin \nu \alpha \sin \nu(\omega-\theta)}{\sin \nu \omega} Q_{\nu-1 / 2}(\cosh \beta) d \nu,
\end{aligned}
$$

where $0<\nu_{1}<\pi / \omega$.

$$
\begin{aligned}
V(\alpha, \beta)= & V^{0}-\quad \text { Case } 2(\theta \leqslant \alpha \leqslant \omega) \\
& V^{0} \sqrt{\frac{\cosh \beta-\cos (\alpha-\theta)}{2}} . \\
& \frac{4}{\pi i} \int_{\nu_{1}-i \infty}^{\nu_{1}+i \infty} \frac{\sin \nu \theta \sin \nu(\omega-\alpha)}{\sin \nu \omega} Q_{\nu-1 / 2}(\cosh \beta) d \nu,
\end{aligned}
$$

where $0<\nu_{1}<\pi / \omega$.

The method of finding the total charge (eq 6.9) on the conductor is based on the general property of the Newtonian potential of any finite distribution of total charge $M$, as in eq 5.4,

$$
\operatorname{limit}_{r \rightarrow \infty}(r V)=M \text {. }
$$

To check the expression (6.9) we may multiply eq 6.10 by $r$ where, as in eq 5.4 ,

$$
r=\frac{c \sqrt{2} \sqrt{\cos (\alpha-\theta)}}{\sqrt{\left(1-c^{2} / r^{2}\right)(\cosh \beta-\cos (\alpha-\theta))}},
$$

which becomes, when $r$ is large ( $\beta$ and $\alpha-\theta$ small),

$r=\frac{c \sqrt{2}}{\sqrt{\cosh \beta-\cos (\alpha-\theta)}}=\frac{c}{\sqrt{\left(\cosh ^{2} \beta / 2-\cos ^{2}\left(\frac{\alpha-\theta}{2}\right)\right.} .}$
This gives

$$
\frac{M}{c V^{0}}=\lim _{\substack{\alpha \rightarrow \theta \\ \beta \rightarrow 0}}\left\{\frac{1}{\sqrt{\cosh ^{2} \frac{\beta}{2}-\cos ^{2}\left(\frac{\alpha-\theta}{2}\right)}}-\right.
$$

$$
\begin{aligned}
& {\left.\left[S_{\omega}(\alpha-\theta, \beta)-S_{\omega}(\alpha+\theta, \beta)\right]\right\} } \\
= & S_{\omega}(2 \theta, 0)-\lim _{\alpha \rightarrow \theta}\left[S_{\omega}(\alpha, 0)-\frac{1}{\sin \alpha / 2}\right] \\
= & S_{\omega}(2 \theta, 0)-G_{\omega}(0,0)+\lim _{\alpha \rightarrow 0} \\
& {\left[\frac{\pi}{\omega \sin \frac{\pi \alpha}{2 \omega}}-\frac{1}{\sin \alpha / 2}\right] }
\end{aligned}
$$

by eq 6.2

$$
=S_{\omega}(2 \theta, 0)-G_{\omega}(0,0),
$$

verifying eq 6.9 .

For general values of $\omega$, the expression (6.9) or $(6.9)^{\prime}$ for total charge cannot be expressed in finite terms. The next section, VII, is concerned with its series expansion and its transformation into a series more suitable for computation.

The direct method of getting $M$ would consist in evaluating separately the charge $M_{1}$, corresponding to the boundary $\alpha=0$ and the charge $M_{2}$ on the remaining boundary $\alpha=\omega$.

Thus

$M_{1}=2 \pi \int \rho \sigma_{1}(\cosh \beta) d s=c^{2} \int_{0}^{\infty} \frac{2 \pi \sigma_{1}(\cosh \beta) \sinh \beta d \beta}{(\cosh \beta-\cos \theta)^{2}}$, or

$$
M_{1}=c^{2} \int_{1}^{\infty} \frac{2 \pi \sigma_{1}(x) d x}{[x-\cos \theta]^{2}},
$$

and similarly,

$$
M_{2}=c^{2} \int_{1}^{\infty} \frac{2 \pi \sigma_{2}(x) d x}{[x-\cos (\omega-\theta)]^{2}},
$$

where, by eq $6.12 \mathrm{a}$,

$2 \pi \sigma_{1}(\cosh \beta)=-\frac{[\cosh \beta-\cos \theta]}{2 c}\left(\frac{\partial V}{\partial \alpha}\right)_{\alpha=0}=\frac{V^{0}}{c}[\cosh \beta-\cos \theta]^{3 / 2} \frac{\sqrt{2}}{\pi i} \int_{\nu_{1}-i \infty}^{\nu_{1}+i \infty} \frac{\nu \sin \nu(\omega-\theta)}{\sin \nu \omega} Q_{\nu-1 / 2}(\cosh \beta) d \nu$

and

$2 \pi \sigma_{2}(\cosh \beta)=+\frac{[\cosh \beta-\cos (\omega-\theta)]}{2 c}\left(\frac{\partial V}{\partial \alpha}\right)_{\alpha=\omega}=\frac{V^{0}}{c}[\cosh \beta-\cos (\omega-\theta)]^{3 / 2} \frac{\sqrt{2}}{\pi i} \int_{\nu_{1}-i \infty}^{\nu_{1}+i \infty} \frac{\nu \sin \nu \theta}{\sin \nu \omega} Q_{\nu-1 / 2}(\cosh \beta) d \nu$.

Using these densities gives

$$
\begin{aligned}
M_{1} & =\frac{c V^{0}}{\pi i} \int_{\nu_{1}-i \infty}^{\nu_{1}+i \infty} \frac{\nu \sin \nu(\omega-\theta)}{\sin \nu \omega} d \nu \int_{1}^{\infty} \frac{\sqrt{2} Q_{\nu-1 / 2}(x)}{\sqrt{x-\cos \theta}} d x \\
& =c V^{0} F(\omega-\theta, \theta)
\end{aligned}
$$

and

$$
\begin{aligned}
M_{2} & =\frac{c V^{0}}{\pi i} \int_{\nu_{1}-i \infty}^{\nu_{1}+i \infty} \frac{\nu \sin \nu \theta}{\sin \nu \omega} \int_{1}^{\infty} \frac{\sqrt{2} Q_{\nu-1_{2}}(x)}{\sqrt{x-\cos (\omega-\theta)}} d x \\
& =c V^{0} F(\theta, \omega-\theta),
\end{aligned}
$$


where

$$
0<\nu_{1}<\pi / \omega
$$

If it were necessary to evaluate $M_{1}$ or $M_{2}$, the $x$-integral in each case is obtained from eq $3.16 \mathrm{~b}$, but in terms of an $s$-series. The integration with respect to $\nu$ could then be performed by deforming the path and evaluating the residues at the poles of the integrand. The $s$-series in eq $3.16 \mathrm{~b}$ would lead ultimately to a double-series in the expression for $F(\omega-\theta, \theta)$. We do not pursue this further as eq 6.9 gives $M$ where

$$
M=M_{1}+M_{2}=c V^{0}[F(\omega-\theta, \theta)+F(\theta, \omega-\theta)] .
$$

\section{Capitance of the Conductor}

If lengths are in centimeters, the capacity $C$ of the conductor is $C_{\omega}(\theta)=M / \mathrm{T}^{0}$ (in centimeters), by eq 6.9 and 1.4 ,

$C_{\omega}(\theta)=a \sin \theta\left\{S_{\omega}(2 \theta, 0)-\left[S_{\omega}(\alpha, 0)-\frac{1}{\sin \alpha / 2}\right]_{\alpha \rightarrow 0}\right\}$

$=a \sin \theta\left[S_{\omega}(2 \theta, 0)-G_{\omega}(0,0)\right]$

$=a \sin \theta\left[\frac{\pi}{\omega \sin \frac{\pi \theta}{\omega}}+G_{\omega}(2 \theta, 0)-G_{\omega}(0,0)\right]$

A formula by which the capacitance could be computed is (from eq 6.7)

$C_{\omega}(\theta)=\frac{a \sin \theta}{\omega}\left\{\frac{\pi}{\sin \frac{\pi \theta}{\omega}}-8 \sum_{n=1}^{\infty} \sin ^{2} \frac{n \pi \theta}{\omega}\left[\psi\left(n+\frac{1}{2}\right)-\right.\right.$

$\left.\left.\psi\left(\frac{n \pi}{\omega}+\frac{1}{2}\right)+\log \frac{\pi}{\omega}\right]\right\}$

where values of the psi-function may be taken from tables such as the six tables, No. 7 to No. 12, of $\psi(x)$ given by Davis. ${ }^{2}$

For actual computations, $C \omega(\theta)$ of eq 7.2 could be expressed as a finite number of terms plus an infinite series of the form

$$
\sum_{n=1}^{\infty} A_{n} \sin ^{2}(n \pi / \omega),
$$

where only a few terms would be required if the coefficients $A_{n}$ vanish with increasing $n$ like, say, $n^{-14}$ instead of like $n^{-2}$ in eq (7.2). To get such a form we may, in eq 7.2, make use of the known asymptotic expansion of the psi-function, valid when $z$ is large with positive real part

$$
\psi(z) \sim \log z-\frac{1}{2 z}+\sum_{t=1}^{N} \frac{(-1)^{t} B_{t}}{2 t z^{2 t}},
$$

where the Bernoulli's numbers are

$$
B_{1}=\frac{1}{6}, B_{2}=\frac{1}{30}, B_{3}=\frac{1}{42}, B_{4}=\frac{1}{30}, B_{5}=\frac{5}{66}, B_{6}=\frac{691}{2730} \text {. }
$$

By use of this it is found by expanding $\psi\left(n+\frac{1}{2}\right)$ and $\psi\left(\frac{n \pi}{\omega}+\frac{1}{2}\right)$ in powers of $1 / n$ that odd powers cancel up to $n^{-11}$, giving

$$
\begin{aligned}
& {\left[\psi\left(n+\frac{1}{2}\right)-\psi\left(\frac{n \pi}{\omega}+\frac{1}{2}\right)+\log \frac{\pi}{\omega}\right] \sim} \\
& \frac{1}{6}\left[\sum_{p=1}^{6}(-1)^{p+1} c_{p}\left[\frac{1-\left(\frac{\omega}{\pi}\right)^{2 p}}{(2 n)^{2 p}}\right]+\text { zero }\left(\frac{1}{n^{14}}\right)\right],
\end{aligned}
$$

where

$$
\left.\begin{array}{l}
c_{1}=1, c_{2}=\frac{7}{10}, c_{3}=\frac{31}{21}, c_{4}=\frac{127}{20} \\
c_{5}=27+\frac{28}{33} \text { and } c_{6}=518+\frac{11}{91}
\end{array}\right\} .
$$

The remainder $\left(n^{-14}\right)$ is less than the last term retained (in this case the term for $p=6$ ). It vanished to higher order than $n^{-12}$ (presumably like $n^{-14}$ ).

If $p$ is a positive integer, the following type of cosine-series converges for

$$
0 \leqslant \alpha \leqslant 2 \omega,
$$

and its sum is a polynominal $h_{2 p}\left(\frac{\pi \alpha}{\omega}\right)$ of degree $2 p$ in $\left(\frac{\pi \alpha}{\omega}\right)$

$$
h_{2 p}\left(\frac{\pi \alpha}{\omega}\right) \equiv \sum_{n=1}^{\infty} \frac{\cos (n \pi \alpha / \omega)}{n^{2 p}}=\sum_{r=0}^{p-1}\left(-1^{r}\right) S_{2 p-2 r} \frac{\left(\frac{\pi \alpha}{\omega}\right)^{2 r}}{(2 r) !}+\frac{(-1)^{p}}{2}\left[\frac{\pi\left(\frac{\pi \alpha}{\omega}\right)^{2 p-1}}{(2 p-1) !}-\frac{\left(\frac{\pi \alpha}{\omega}\right)^{2^{p}}}{(2 p) !}\right],
$$

\footnotetext{
${ }^{2}$ H. T. Davis, Tables of the higher mathernatical functions, I (Principia Press, Bloomington, Indiana, 1933).
} 
where

$$
\begin{gathered}
S_{2 p}=\frac{(2 \pi)^{2 p}}{2(2 p) !} B_{p}=\sum_{s=1}^{\infty} \frac{1}{s^{2 p}} \\
S_{2}=1.644934 \\
S_{4}=1.082323 \\
S_{6}=1.017343 \\
S_{8}=1.004077 \\
S_{10}=1.000995
\end{gathered}
$$

With the second member of eq 7.5 we could form series whose sum represents a polynominal in $(\pi \alpha / \omega)$ of 12 th degree, which could be computed without the necessity of computing the Fourier series, let

$H\left(\frac{\pi \alpha}{\omega}\right) \equiv \frac{1}{6} \sum_{p=1}^{6}(-1)^{p+1} c_{p}\left[\frac{1-\left(\frac{\omega}{\pi}\right)^{2 p}}{2^{2 p}}\right] h_{2 p}\left(\frac{\pi \alpha}{\omega}\right)$.

This may be written as the sum of 6 Fourier series

$$
\begin{aligned}
H\left(\frac{\pi \alpha}{\omega}\right)= & \frac{1}{6} \sum_{p=1}^{6}(-1)^{p+1} c_{p}\left[\frac{1-\left(\frac{\omega}{\pi}\right)^{2 p}}{2^{2 p}}\right] \\
& \sum_{n=1}^{\infty} \frac{\cos (n \pi \alpha / \omega)}{n^{2 p}} .
\end{aligned}
$$

Equation 6.7 is

$$
\begin{aligned}
G_{\omega}(\alpha, 0)= & \frac{4}{\omega}\left\{\frac{1}{2} \log \frac{\pi}{\omega}+\sum_{n=1}^{\infty} \cos \left(\frac{n \pi \alpha}{\omega}\right)\right. \\
& {\left.\left[\psi\left(n+\frac{1}{2}\right)-\psi\left(\frac{n \pi}{\omega}+\frac{1}{2}\right)+\log \frac{\pi}{\omega}\right]\right\} } \\
= & \frac{4}{\omega}\left\{\frac{1}{2} \log \frac{\pi}{\omega}+H\left(\frac{\pi \alpha}{\omega}\right)+\sum_{n=1}^{\infty} A_{n} \cos \frac{n \pi \alpha}{\omega}\right\},
\end{aligned}
$$

where

$$
\begin{aligned}
A_{n}= & {\left[\psi\left(n+\frac{1}{2}\right)-\psi\left(\frac{n \pi}{\omega}+\frac{1}{2}\right)+\log \frac{\pi}{\omega}\right]-} \\
& \frac{1}{6} \sum_{p=1}^{6}(-1)^{p+1} c_{p}\left[\frac{1-\left(\frac{\omega}{\pi}\right)^{2 p}}{(2 n)^{2 p}}\right] .
\end{aligned}
$$

For large $n$, eq 7.4 becomes

$$
A_{n} \sim \frac{1}{6} \text { Zero }\left(\frac{1}{n^{14}}\right) .
$$

Hence, very few terms of the infinite series in eq 7.8 will be sufficient.
Formula (7.1) for capacitance becomes

$$
\begin{aligned}
C_{\omega}(\theta)= & \frac{a \sin \theta}{\omega}\left\{\frac{\pi}{\sin \frac{\pi \theta}{\omega}}-4\left[H(0)-H\left(\frac{2 \pi \theta}{\omega}\right)\right]-\right. \\
& \left.8 \sum_{n=1}^{\infty} A_{n} \sin ^{2} \frac{n \pi}{\omega}\right\} .
\end{aligned}
$$

The polynominal $H\left(\frac{2 \pi \theta}{\omega}\right)$ may be computed by eq 7.7 and $7.5, \alpha$ being replaced by $2 \theta$ and again by zero for $H(0)$. This formula (7.9) is the exact equivalent of eq 7.2. It becomes an approximation when the infinite series in eq 7.9 is replaced by the first two or three terms, but it gives a much closer approximation than the same number of terms of the series (7.2). Formulas for $C$ will be found below, in finite terms, when $\pi / \omega$ rational say $\omega=n \pi / n$, but if either of the integers $n$ or $m$ is large, the new formulas may not be preferable to eq 7.9 for numerical computation.

The capacitance of a conductor that consists of two unequal spheres in external contact may be computed by this method.

Let

$$
b \equiv \frac{2 a_{1}}{a+a_{1}} \text { where } 0<a_{1} \leqslant a, \text { and } 0<b \leqslant 1 .
$$

Equation 1.2 shows that $\theta$ and $\omega$ each approach zero, their ratio being

$$
\frac{\theta}{\omega}=\frac{b}{2}
$$

In this case the capacitance is

$$
\begin{aligned}
C_{0}(0)= & \frac{a a_{1}}{a+a_{1}}\left\{\frac{\pi}{\sin \pi b / 2}-4[H(0)-H(\pi b)]-\right. \\
& \left.8 \sum_{n=1}^{\infty} A_{n} \sin ^{2} \frac{n \pi b}{2}\right\},
\end{aligned}
$$

where

$$
\begin{gathered}
H(\pi b)=\frac{1}{6} \sum_{p=1}^{6}(-1)^{p+1} \frac{c_{p}}{4^{p}} h_{2 p}(\pi b), \\
h_{2 p}(\pi b)=\sum_{r=0}^{p-1}(-1)^{r} \frac{(\pi b)^{2 r}}{(2 r) !}+\frac{(-1)^{p}}{2}\left(\pi-\frac{\pi b}{2 p}\right) \frac{(\pi b)^{2 p-1}}{(2 p-1) !}
\end{gathered}
$$


and

$A_{n}=\psi\left(n+\frac{1}{2}\right)-\log n-\frac{1}{6} \sum_{p-1}^{6} \frac{(-1)^{p+1} c_{p}}{(2 n)^{2 p}}$,

so $A_{n} \sim$ Zero $\left(\frac{1}{n^{14}}\right)$ when $n$ is large.

For equal spheres $b=1$.

\section{Capacitance in Finite Terms $(\omega / \pi$ rational)}

By eq 4.12, in which $\beta$ and $\beta^{\prime}$ are each zero and $\omega=n \pi$,

$$
S_{n \pi / m}(\alpha, 0)=\sum_{t=0}^{m-1} S_{n \pi}\left(\alpha+\frac{2 n \pi t}{m}, 0\right) .
$$

Hence, to obtain $S_{\omega}(\alpha, 0)$ when $\omega=n \pi / m$, we require expressions for $S_{n \pi}(\alpha, 0)$. These are found from eq $6.1 \mathrm{c}$ with $\beta=0$. It is necessary to distinguish between even and odd $n$.

For odd, write $\omega=(2 n-1) \pi$, and for even take $\omega=2 n \pi$ where $n$ is a positive integer in each case. Equation $6.1 \mathrm{c}$ gives

$S_{(2 n-1) \pi}(\alpha, 0)=$

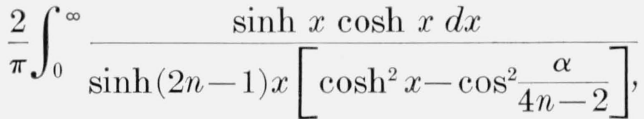

in which we take $\alpha$ in the period interval,

$$
0<\alpha<2 \omega=(4 n-2) \pi \text { so } 0<\frac{\alpha}{4 n-2}<\pi .
$$

Similarly,

$S_{2 n \pi}(\alpha, 0)=\frac{2}{\pi} \int_{0}^{\infty} \frac{\sinh x \cosh x d x}{\sinh 2 n x\left[\cosh ^{2} x-\cos ^{2} \frac{\alpha}{4 n}\right]}$,

where $\alpha$ is in the period interval,

$$
0<\alpha<2 \omega=4 n \pi \text { so } 0<\frac{\alpha}{4 n}<\pi \text {. }
$$

These may be integrated by use of the substitution $t=\cosh x$, if we make use of the finite products that are valid for real or complex $x$, and $n>1$

$$
\frac{\sinh (2 n-1) x}{\sinh x}=(2 n-1) \prod_{s=1}^{n-1} \frac{\cosh ^{2} x-\cos ^{2} \frac{s \pi}{2 n-1}}{\sin ^{2} \frac{s \pi}{2 n-1}}
$$

$\frac{\sinh 2 n x}{\sinh x \cosh x}=2 n \prod_{s=1}^{n-1} \frac{\cosh ^{2} x-\cos ^{2} \frac{s \pi}{2 n}}{\sin ^{2} \frac{s \pi}{2 n}}$.

Considering these ratios as polynominals in $t=\cosh x$, we find the following resolution into partial fractions for their reciprocals,

$$
\begin{aligned}
& \frac{\sinh x}{\sinh (2 n-1) x}= \\
& \frac{2}{2 n-1} \sum_{s=1}^{n-1} \frac{(1-)^{s+1} \sin ^{2}\left(\frac{s \pi}{2 \mathrm{n}-1}\right) \cos \left(\frac{s \pi}{2 n-1}\right)}{\cosh ^{2} x-\cos ^{2}\left(\frac{s \pi}{2 n-1}\right)}
\end{aligned}
$$

$$
\frac{\sinh x \cosh x}{\sinh 2 n x}=\frac{1}{4 n} \sum_{s=1}^{n-1} \frac{(-1)^{s+1} \sin ^{2}\left(\frac{s \pi}{n}\right)}{\cosh ^{2} x-\cos ^{2}\left(\frac{s \pi}{2 n}\right)}
$$

For $n>1$ the integrals (eq 8.1a, b) become

$$
\begin{array}{r}
S_{(2 n-1) \pi}(\alpha, 0)=\frac{2}{2 n-1} \sum_{s=1}^{n-1} \frac{(-1)^{s+1} \sin ^{2} \frac{s \pi}{2 n-1} \cos \left(\frac{s \pi}{2 n-1}\right)}{\cos ^{2} \frac{s \pi}{2 n-1}-\cos ^{2} \frac{\alpha}{4 n-2}} . \\
\frac{2}{\pi} \int_{1}^{\infty}\left[\frac{t}{t^{2}-\cos ^{2} \frac{s \pi}{2 n-1}}-\frac{t}{t^{2}-\cos ^{2} \frac{\alpha}{4 n-2}}\right] \frac{d t}{\sqrt{t^{2}-1}},
\end{array}
$$

$$
\begin{aligned}
S_{2 n \pi}(\alpha, 0) & =\frac{1}{4 n} \sum_{s=1}^{n-1} \frac{(-1)^{s+1} \sin ^{2} \frac{s \pi}{4 n}}{\cos ^{2} \frac{s \pi}{2 n}-\cos ^{2} \frac{\alpha}{4 n}} . \\
& \frac{2}{\pi} \int_{1}^{\infty}\left[\frac{1}{t^{2}-\cos ^{2} \frac{s \pi}{2 n}}-\frac{1}{t^{2}-\cos ^{2} \frac{\alpha}{4 n}}\right] \frac{d t}{\sqrt{t^{2}-1}} .
\end{aligned}
$$

Letting $t=1 / \cos \phi$, the integrals in eq $8.4 \mathrm{a}$ and $8.4 \mathrm{~b}$ become $I_{s}^{(a)}$ and $I_{s}^{(b)}$, where

$$
\begin{aligned}
I_{s}^{(a)}= & \frac{1}{\pi} \int_{0}^{\pi / 2}\left[\frac{1}{1-\cos \frac{s \pi}{2 n-1} \cos \phi}+\right. \\
& \frac{1}{1+\cos \frac{s \pi}{2 n-1} \cos \phi}-\frac{1}{1-\cos \frac{\alpha}{4 n-2} \cos \phi} \\
& \left.\frac{1}{1+\cos \frac{\alpha}{4 n-2} \cos \phi}\right] d \phi,
\end{aligned}
$$




$$
\begin{aligned}
I_{s}^{(b)}= & \frac{1}{\pi} \int_{0}^{\pi / 2}\left[\frac { 1 } { \operatorname { c o s } \frac { s \pi } { 2 n } } \left(\frac{1}{1-\cos \frac{s \pi}{2 n} \cos \phi}-\right.\right. \\
& \left.\frac{1}{1+\cos \frac{s \pi}{2 n} \cos \phi}\right)-\frac{1}{\cos \frac{\alpha}{4 n}} \cdot \\
& \left.\left(\frac{1}{1-\cos \frac{\alpha}{4 n} \cos \phi}-\frac{1}{1+\cos \frac{\alpha}{4 n} \cos \phi}\right)\right] d \phi .
\end{aligned}
$$

Now

$$
\begin{aligned}
& \begin{array}{l}
\frac{1}{\pi} \int_{0}^{\pi / 2} \frac{d \phi}{1+\cos \gamma \cos \phi}=\frac{\gamma}{\pi \sin \gamma} \text { if } 0 \leqslant \gamma<\pi, \\
\frac{1}{\pi} \int_{0}^{\pi / 2} \frac{d \phi}{1-\cos \gamma \cos \phi}=\frac{\pi-\gamma}{\pi \sin \gamma} \text { if } 0<\gamma<2 \pi, \\
\frac{2}{\pi} \int_{0}^{\pi / 2} \frac{d \phi}{1-\cos ^{2} \gamma \cos ^{2} \phi}=\frac{1}{\sin \gamma} \text { if } 0<\gamma<\pi, \\
\frac{1}{\pi} \int_{0}^{\pi / 2} \frac{\cos \phi d \phi}{1-\cos ^{2} \gamma \cos ^{2} \phi}=\frac{1-2 \gamma / \pi}{\sin 2 \gamma} \text { if } 0<\gamma<\pi,
\end{array} \\
& \begin{array}{l}
\frac{1}{\pi} \int_{0}^{\pi / 2} \frac{d \phi}{1+\cos \gamma \cos \phi}=\frac{\gamma}{\pi \sin \gamma} \text { if } 0 \leqslant \gamma<\pi, \\
\frac{1}{\pi} \int_{0}^{\pi / 2} \frac{d \phi}{1-\cos \gamma \cos \phi}=\frac{\pi-\gamma}{\pi \sin \gamma} \text { if } 0<\gamma<2 \pi, \\
\frac{2}{\pi} \int_{0}^{\pi / 2} \frac{d \phi}{1-\cos ^{2} \gamma \cos ^{2} \phi}=\frac{1}{\sin \gamma} \text { if } 0<\gamma<\pi, \\
\frac{1}{\pi} \int_{0}^{\pi / 2} \frac{\cos \phi d \phi}{1-\cos ^{2} \gamma \cos ^{2} \phi}=\frac{1-2 \gamma / \pi}{\sin 2 \gamma} \text { if } 0<\gamma<\pi,
\end{array} \\
& \begin{array}{l}
\frac{1}{\pi} \int_{0}^{\pi / 2} \frac{d \phi}{1+\cos \gamma \cos \phi}=\frac{\gamma}{\pi \sin \gamma} \text { if } 0 \leqslant \gamma<\pi, \\
\frac{1}{\pi} \int_{0}^{\pi / 2} \frac{d \phi}{1-\cos \gamma \cos \phi}=\frac{\pi-\gamma}{\pi \sin \gamma} \text { if } 0<\gamma<2 \pi, \\
\frac{2}{\pi} \int_{0}^{\pi / 2} \frac{d \phi}{1-\cos ^{2} \gamma \cos ^{2} \phi}=\frac{1}{\sin \gamma} \text { if } 0<\gamma<\pi, \\
\frac{1}{\pi} \int_{0}^{\pi / 2} \frac{\cos \phi d \phi}{1-\cos ^{2} \gamma \cos ^{2} \phi}=\frac{1-2 \gamma / \pi}{\sin 2 \gamma} \text { if } 0<\gamma<\pi,
\end{array} \\
& \begin{array}{l}
\frac{1}{\pi} \int_{0}^{\pi / 2} \frac{d \phi}{1+\cos \gamma \cos \phi}=\frac{\gamma}{\pi \sin \gamma} \text { if } 0 \leqslant \gamma<\pi, \\
\frac{1}{\pi} \int_{0}^{\pi / 2} \frac{d \phi}{1-\cos \gamma \cos \phi}=\frac{\pi-\gamma}{\pi \sin \gamma} \text { if } 0<\gamma<2 \pi, \\
\frac{2}{\pi} \int_{0}^{\pi / 2} \frac{d \phi}{1-\cos ^{2} \gamma \cos ^{2} \phi}=\frac{1}{\sin \gamma} \text { if } 0<\gamma<\pi, \\
\frac{1}{\pi} \int_{0}^{\pi / 2} \frac{\cos \phi d \phi}{1-\cos ^{2} \gamma \cos ^{2} \phi}=\frac{1-2 \gamma / \pi}{\sin 2 \gamma} \text { if } 0<\gamma<\pi,
\end{array}
\end{aligned}
$$

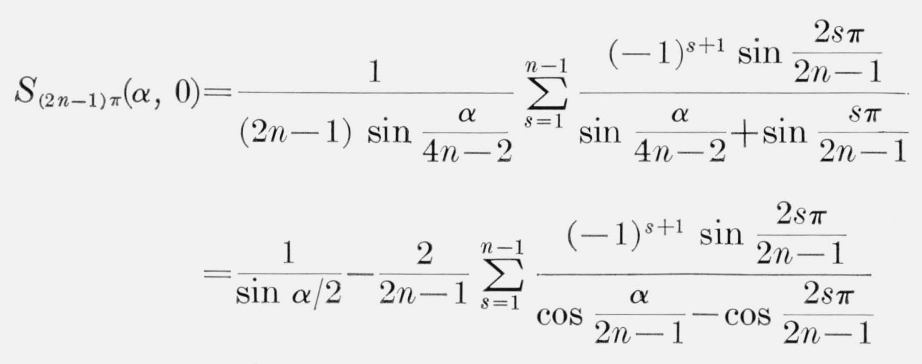$$
I_{s}^{(\alpha)}=\frac{1}{\sin \frac{s \pi}{2 n-1}}-\frac{1}{\sin \frac{\alpha}{4 n-2}}
$$$$
I_{s}^{(b)}=2\left[\frac{1-\frac{s}{n}}{\sin \frac{s \pi}{n}}-\frac{1-\frac{\alpha}{2 \pi n}}{\sin \frac{\alpha}{2 n}}\right] \text {. }
$$

The formulas obtained, which apply throughout the fundamental period interval $0<\alpha<2 \pi$ (boundaries excluded where $\left.S_{\omega}(\alpha, 0) \rightarrow \infty\right)$, are

$$
S_{\pi}(\alpha, 0)=\frac{1}{\sin \alpha / 2} \text { for } 0<\alpha<2 \pi .
$$

For $n>1$ and $0<\alpha<2 \omega=(2 n-1) 2 \pi$, we find

$$
S_{2 \pi}(\alpha, 0)=\frac{1-\frac{\alpha}{2 \pi}}{\sin \alpha / 2} \text { for } 0<\alpha<2 \omega=4 \pi .
$$

For $n>1$ and $0<\alpha<2 \omega=4 n \pi$, it is found that

$$
\begin{aligned}
& S_{2 n \pi}(\alpha, 0)=\frac{1}{n} \sum_{s=1}^{n-1} \frac{(-1)^{s+1} \sin ^{2} \frac{s \pi}{n}}{\cos \frac{\alpha}{2 n}-\cos \frac{s \pi}{n}}\left[\frac{1-\frac{\alpha}{2 n \pi}}{\sin \alpha / 2 n}-\frac{1-\frac{s}{n}}{\sin s \pi / n}\right] \\
& =\frac{1-\frac{\alpha}{2 n \pi}}{\sin \alpha / 2}-\frac{1}{n} \sum_{s=1}^{n-1} \frac{(-1)^{s+1}\left(1-\frac{s}{n}\right) \sin \frac{s \pi}{n}}{\cos \frac{\alpha}{2 n}-\cos \frac{s \pi}{n}}
\end{aligned}
$$

by eq $8.3 b$ with $x=i \alpha / 4 n$ ). If $\alpha$ were replaced by $|\alpha|$, these expressions would all serve for period interval $-\omega<\alpha<\omega$. It is readily verified that these expressions for $S_{n \pi}(\alpha, 0)$ satisfy the condition of symmetry,

$S_{\omega}(\alpha, 0)=S_{\omega}(2 \omega-\alpha, 0)$ so that $D_{\alpha} S_{\omega}(\alpha, 0)=0$ when $\alpha=\omega$.
Since eq 8.5 to $8.7^{\prime}$ are all valid for the periodinterval, $0<\alpha<2 \omega$, these may be used in eq 4.12 to obtain $S_{n \pi / m}(\alpha, 0)$ for its positive period interval.

The second form (eq 8.5)' is used only to get $G_{\omega}(0,0)=\left[S_{\omega}(\alpha, 0)-\frac{1}{\sin \alpha / 2}\right]$ for $\alpha=0$. The same remark applies for eq $8.7^{\prime}$. 
The capacitance is given by eq 7.1, in which the most suitable form to be used here is

$$
C_{\omega}(\theta)=a \sin \theta\left[S_{\omega}(2 \theta, 0)-G_{\omega}(0,0)\right] .
$$

In the formulas for capacitance given below it is understood that $\theta$ satisfies the two inequalities of eq 1.1, namely,
Consequently, $0<2 \theta<2 \omega$, that is $2 \theta$ is in the first positive period-interval.

$$
C_{\pi / m}(\theta)=a+a \sin \theta \sum_{t=1}^{m-1}\left[\frac{1}{\sin \left(\frac{t \pi}{m}+\theta\right)}-\frac{1}{\sin \frac{t \pi}{m}}\right],
$$

where $m>1$.

For $\omega=\frac{(2 n-1) \pi}{2 m}<2 \pi$, where $1<n \leqslant 2 m$,

$$
\begin{aligned}
& C_{\frac{(2 n-1) \pi}{2 m}}(\theta)=\frac{a \sin \theta}{2 n-1}\left\{\sum_{s=1}^{m-1} \sum_{t=0}^{2 m-1} \frac{(-1)^{s+1} \sin \frac{2 s \pi}{2 n-1}}{\sin \left(\frac{t \pi}{2 m}+\frac{\theta}{2 n-1}\right)\left[\sin \left(\frac{t \pi}{2 m}+\frac{\theta}{2 n-1}\right)+\sin \frac{s \pi}{2 n-1}\right]^{+}}\right. \\
& 2 \sum_{s=1}^{n-1} \sum_{t=0}^{2 m-1} \frac{(-1)^{s+1} \sin \frac{2 s \pi}{2 n-1}}{\cos \frac{t \pi}{m}-\cos \frac{2 s \pi}{2 n-1}}-\left(2 n-1 \sum_{t=1}^{2 m-1} \frac{1}{\sin \frac{(2 n-1) t \pi}{2 m}}\right\} \\
& C_{2 \pi}(\theta)=a-\frac{a}{\pi}(\theta-\sin \theta) \\
& C_{2 \pi / m}(\theta)=a-\frac{a}{\pi}(\theta-\sin \theta)+a \sin \theta \sum_{t=1}^{m-1}\left[\frac{1-\left(\frac{2 t}{m}+\frac{\theta}{\pi}\right)}{\sin \left(\frac{2 \pi t}{m}+\theta\right)}-\frac{1-\frac{2 t}{m}}{\sin 2 \pi t / m}\right],
\end{aligned}
$$

where $m>2$ (fig. 1, a).

For $\omega=\frac{2 n \pi}{2 m-1}<2 \pi$ where $1<n<2 m-1$,

$$
\begin{aligned}
C_{\frac{2 n \pi}{2 m-1}}(\theta)= & \frac{a \sin \theta}{n}\left\{\sum_{s=1}^{n-1} \sum_{t=0}^{2 m-2}\left[\cos \left(\frac{2 t \pi}{2 m-1}+\frac{\theta}{n}\right)-\cos \frac{s \pi}{n}\right]\left[\frac{1-\frac{1}{\pi}\left(\frac{2 t \pi}{2 m-1}+\frac{\theta}{n}\right)}{\sin \left(\frac{2 t \pi}{2 m-1}+\frac{\theta}{n}\right)}-\frac{1-s / n}{\sin s \pi / n}\right]+\right. \\
& \left.\sum_{s=1}^{n-1} \sum_{t=0}^{2 m-2} \frac{(-1)^{s+1}\left(1-\frac{s}{n}\right) \sin \frac{s \pi}{n}}{\cos \frac{2 t \pi}{2 m-1}-\cos \frac{s \pi}{n}}+\frac{1}{\pi}-n \sum_{t=1}^{2 m-2} \frac{1-\frac{2 t}{2 m-1}}{\sin \left(\frac{2 n t \pi}{2 m-1}\right)}\right\}
\end{aligned}
$$

The case $\omega=2 \pi$ is a thin spherical shell with a hole of angular aperture $2 \theta$. The capacitance is given in eq 18.11. When the conductor is formed by two unequal spheres intersecting orthogonally, there are two cases, $\omega=\pi / 2$ and $\omega=3 \pi / 2$. The first equation (8.8) with $m=2$ gives the capacitance, for $0<\theta<\pi / 2$, (see a,b of fig. 1)

$$
C_{\pi / 2}(\theta)=a+a \tan \theta-a \sin \theta=a+a_{1}-\frac{a a_{1}}{\sqrt{a^{2}+a_{1}^{2}}},
$$

the radius $a_{1}$ being $a$ tan $\theta$ by eq 1.2 .

The other case $\omega=3 \pi / 2$ is given by eq 8.9 with $n=2$ and $m=1$,

$$
C_{3 \pi / 2}(\theta)=\frac{a \sin \theta}{\sqrt{3}}\left[\sqrt{3}-\frac{4}{3}+\frac{1}{2 \sin \frac{\theta}{3}\left(\sin \frac{\theta}{3}+\sin \frac{\pi}{3}\right)}+\frac{1}{2 \cos \frac{\theta}{3}\left(\cos \frac{\theta}{3}+\sin \frac{\pi}{3}\right)}\right] .
$$


When $\theta=\pi / 2$, this conductor is a hemisphere, the face $\alpha=\omega=3 \pi / 2$ is a plane face. This gives

$$
C_{3 \pi / 2}(\pi / 2)=\frac{2 a}{\sqrt{3}}(\sqrt{3}-1)=.8453 a,
$$

as the capacitance of the hemisphere of radius $a$. (Fig. 1, d). Placing $\theta=\pi / 2$ in eq 8.11 gives

$$
C_{2 \pi}(\pi / 2)=a\left(\frac{1}{2}+\frac{1}{\pi}\right)=.8183 a
$$

as the capacitance of a hemispherical bowl.

In general when $\omega>\pi$, as it may be in eq 8.9 or 8.12 , we may then take $\theta=\omega-\pi$, in which case the conductor has one plane boundary as in figure $1, \mathrm{c}, \mathrm{d}$.

\section{Electric Field in Finite Terms}

The only case in which the field, that is, $S_{\omega}$ $\left(\alpha, \beta, \beta^{\prime}\right)$, has been found in finite form where neither $\beta$ or $\beta^{\prime}$ is zero, has been given in eq 4.12 with $\omega=\pi / m$. This means that the potential due to any circular line-charge in the presence of the conductor at zero potential is found at all points in space in terms of a finite number of complete elliptic integrals, for the case of an infinite set of values of the form ${ }^{3} \omega=\pi / m, m$ being any positive integer.

When $\beta^{\prime}=0$, we may find three other infinite sets corresponding to $\omega=n \pi / m$ where $n=2,3$, or 4 . These give the electric field in finite terms due to the charged conductor alone, or to the conductor under influence of a point-charge on the $x$-axis. It is merely a question of evaluating $S_{n \pi}(\alpha, \beta)$, that is, $S_{n \pi}(\alpha, \beta, 0)$ for $n=1,2,3,4$.

To do this, place $\omega=n \pi$ in eq $6.1 \mathrm{~b}$,

$$
S_{n \pi}(\alpha, \beta)=\frac{\sqrt{2}}{\pi} \int_{\beta / n}^{\infty} \frac{\sinh x d x}{\left(\cosh x-\cos _{n}^{\alpha}\right) \sqrt{\cosh n x-\cosh \beta}} .
$$

Using the finite product,

$\cosh n x-\cosh \beta=2^{n-1} \Pi_{t=0}^{n-1}\left[\cosh x-\cos \left(\frac{2 t \pi+i \beta}{n}\right)\right]$,

3 The potential was given for the case $\omega=\pi / m$ by H. M. Macdonald, Proc London Math. Soc., (1), XXVI, p. 156 (1895). gives

$S_{n \pi}(\alpha, \beta)=\frac{2}{\pi 2^{n / 2}}$.

$\int_{\beta / n}^{\infty} \frac{\sinh x d x}{\left(\cosh x-\cos \frac{\alpha}{n}\right) \sqrt{\pi_{t=0}^{n-1}\left[\cosh x-\cosh \left(\frac{\beta-2 \pi t i}{n}\right)\right]}} \cdot$

Let

$$
\left.\begin{array}{l}
x^{\prime}=\cosh x \\
x_{0}=\cosh \beta / n \text { and } x_{t}=\cosh \left(\frac{\beta}{n}-\frac{2 \pi t i}{n}\right)
\end{array}\right\}
$$

where $t=1,2,3, \ldots(n-1)$.

This becomes

$$
\begin{aligned}
& S_{n \pi}(\alpha, \beta)=\frac{2}{\pi 2^{n / 2}} \cdot \\
& \int_{x_{0}}^{\infty} \frac{d x^{\prime}}{\left(x^{\prime}-\cos \frac{\alpha}{n}\right) \sqrt{\left(x^{\prime}-x_{0}\right)\left(x^{\prime}-x_{1}\right) \ldots\left(x^{\prime}-x_{n-1}\right)}} .
\end{aligned}
$$

Letting $x^{\prime}=x_{0} x$, this becomes

$S_{n \pi}(\alpha, \beta)=\frac{2}{\pi\left(2 x_{0}\right)^{n / 2}}$

$\int_{1}^{\infty} \frac{d x}{\left(x-\frac{\cos (\alpha / n)}{x_{0}}\right) \sqrt{(x-1)\left(x-\frac{x_{1}}{x_{0}}\right) \cdots\left(x-\frac{x_{n-1}}{x_{0}}\right)}}$.

For $n=1$ and $n=2$, this integrates with elementary functions. When $n=3$ and when $n=4$, it integrates with elliptic functions. For $n \geqslant 5$ this integral is hyperelliptic. For $n=1$ we obtain

$$
S_{\pi}(\alpha, \beta)=\frac{\sqrt{2}}{\sqrt{\cosh \beta-\cos \alpha}}
$$

as before.

The expression for $S_{\pi / n}(\alpha, \beta)$ is given in eq 4.13.

$$
\text { The case } n=2 \text { or } \omega=2 \pi
$$

Equation 9.3 gives $x_{0}=\cosh \beta / 2$ and $x_{1}=-x_{0}$, so eq 9.4 becomes

$$
S_{2 \pi}(\alpha, \beta)=\frac{1}{x_{0} \pi} \int_{1}^{\infty} \frac{d x}{(x-\cos \gamma) \sqrt{x^{2}-1}},
$$


where

$$
\left.\begin{array}{r}
\cos \gamma=\frac{\cos \alpha / 2}{\cosh \beta / 2} \text { and } \sin \gamma=\frac{\sqrt{\cosh \beta-\cos \alpha}}{\sqrt{2} \cdot \cosh \beta / 2} \\
0<\gamma \leqslant \pi \text { when } 0<\alpha / 2 \leqslant \pi
\end{array}\right\},
$$

and when $\beta \rightarrow 0, \gamma \rightarrow \alpha / 2$ for $0<\alpha / 2 \leqslant \pi$.

It is sufficient to restrict $\alpha$ to the half-period interval

$$
0<\alpha \leqslant \omega=2 \pi
$$

The substitution $x=1 / \cos \phi$ gives

$$
S_{2 \pi}(\alpha, \beta)=\frac{1}{x_{0} \pi} \int_{0}^{\pi / 2} \frac{d \phi}{1-\cos \gamma \cos \phi}=\frac{1-\frac{\gamma}{\pi}}{x_{0} \sin \gamma},
$$

or

$S_{2 \pi}(\alpha, \beta)=\frac{\sqrt{2}}{\sqrt{\cosh \beta-\cos \alpha}}\left[1-\frac{1}{\pi} \cos ^{-1}\left(\frac{\cos \alpha / 2}{\cosh \beta / 2}\right)\right]$,

which reduces to eq 8.6 when $\beta=0$.

It may be noticed that the factor of the bracket is $S_{\pi}(\alpha, \beta)$. This appears in the two following forms.

$$
\text { The case } n=3 \text { or } \omega=3 \pi
$$

Consider $\alpha$ in the half-period interval $0<\alpha \leqslant \omega=$ $3 \pi$ so $0<\alpha / 3 \leqslant \pi$. The modulus $k$ is defined by

$$
2 k^{2}=1+\sqrt{3} \frac{\cosh (\beta / 3)}{\sqrt{4 \cosh ^{2}(\beta / 3)-1}} .
$$

Let $\gamma$ (in the interval $0<\gamma<2 K$ ) be determined by

$$
c n \gamma=\frac{\sqrt{3\left(\cosh ^{2} \frac{\beta}{3}-\frac{1}{4}\right)}-(\cosh \beta / 3-\cos \alpha / 3)}{\sqrt{3\left(\cosh ^{2} \frac{\beta}{3}-\frac{1}{4}\right)}+(\cosh \beta / 3-\cos \alpha / 3)} .
$$

It is found that

$S_{3 \pi}(\alpha, \beta)=$

$\frac{\sqrt{2}}{\sqrt{\cosh \beta-\cos \alpha}}\left\{1+\frac{2 K^{\prime}}{\nu}\left[\frac{s n \gamma d n \gamma}{1+c n \gamma}-Z(\gamma)\right]-\frac{\gamma}{K}\right\}$,

where $Z(\gamma)$ is Jacobis zeta-function,

$$
Z(u)=\int_{0}^{u} d n^{2} u^{\prime} d \mu^{\prime}-u \frac{E}{K}=E(u)-u \frac{E}{K} .
$$

The quarter-period for modulus $k$ is $K$ while $K^{\prime}$ is that for complimentary modulus $k^{\prime}=\sqrt{1-k^{2}}$

When $\beta \rightarrow 0$

$$
k \rightarrow 1, K^{\prime} \rightarrow \frac{\pi}{2}, K \rightarrow \infty
$$

$c n \gamma \rightarrow d n \gamma \rightarrow \operatorname{sech} \gamma=\frac{\frac{1}{2}+\cos \frac{\alpha}{3}}{\frac{5}{2}-\cos \frac{\alpha}{3}}>0$ if $0<\alpha<\pi$

$\operatorname{sn} \gamma \rightarrow Z(\gamma) \rightarrow \tanh \gamma$

so

$$
\begin{aligned}
& \frac{2 K^{\prime}}{\pi}\left[\frac{\operatorname{sn} \gamma d n \gamma}{1+\operatorname{cn} \gamma}-Z(\gamma)\right] \rightarrow \frac{-\tanh \gamma}{1+\operatorname{sech} \gamma}=- \\
& \sqrt{\frac{1-\operatorname{sech} \gamma}{1+\operatorname{sech} \gamma}}=-\frac{\sin (\alpha / 6)}{\sin \pi / 3}=-\frac{2}{\sqrt{3}} \sin \frac{\alpha}{6} .
\end{aligned}
$$

Hence eq $9.7 \mathrm{c}$ becomes

$$
\begin{aligned}
S_{3 \pi}(\alpha, 0)= & \frac{1}{\sin \frac{\alpha}{2}}-\frac{2 \sin (\alpha / 6)}{\sqrt{3} \sin \alpha / 2}=\frac{1}{\sin \frac{\alpha}{2}}- \\
& \frac{1}{\sqrt{3}\left(\cos \frac{\alpha}{3}-\cos \frac{2 \pi}{3}\right)}
\end{aligned}
$$

in agreement with eq $8.5^{\prime}$ (with $n=2$ ).

The case $n=4$ or $\omega=4 \pi$

Consider $\alpha$ in the half-period interval $0<\alpha \leqslant$ $\omega=4 \pi$, so $0<\alpha / 4 \leqslant \pi$.

Let

and

$$
k^{2}=\frac{\cosh ^{2} \beta / 4}{\cosh \beta / 2}
$$

$$
c n \gamma=\frac{\cos \alpha / 4}{\cosh \beta / 4} \text { where } 0<\gamma<2 K \text {. }
$$

It is found that

$$
\begin{aligned}
S_{4 \pi}(\alpha, \beta) & =\frac{\sqrt{2}}{\sqrt{\cosh \beta-\cos \alpha}} . \\
& {\left[1-\frac{1}{\pi} \sin ^{-1}(k \sin \gamma)-\frac{K^{\prime}}{\pi} Z(\gamma)-\frac{\gamma}{2 K}\right] . }
\end{aligned}
$$

When $\beta \rightarrow 0$, this gives for $0<\alpha / 4<\pi / 2$,

$$
\begin{gathered}
k \rightarrow 1, K^{\prime} \rightarrow \frac{\pi}{2}, K \rightarrow \infty, \text { cn } \gamma \rightarrow \operatorname{sech} \gamma=\cos \alpha / 4 \\
\operatorname{sn} \gamma \rightarrow Z(\gamma) \rightarrow \tanh \gamma=\sin \alpha / 4 .
\end{gathered}
$$


Therefore,

$S_{4 \pi}(\alpha, 0)=\frac{1-\frac{\alpha}{4 \pi}}{\sin \alpha / 2}-\frac{1}{4 \cos \alpha / 4}$ as in (8.7) with $n=2$.

Hence by eq 4.12 the potential or electric field may be found in finite terms when $\omega=n \pi / m$ where $n=1,2,3$, or 4 and $m$ is any positive integer.

\section{Summary of Formulas for Computing Capacitance and Field}

The conductor has a meridian section bounded by the two ares $\alpha=0$ and $\alpha=\omega$ of figure 2, a. Several sections are shown in figure 1 . The capacitance is a function $C_{\omega}(\theta)$ of the two angles $\omega$ and $\theta$. In case $\theta=\omega-\pi$, the conductor has one plane boundary.

For general values of $\omega$ the capacitance may be computed by eq 7.2 or by its equivalent but more rapidly converging series (7.9).
This method is applicable to the case of two unequal (or equal) spheres in external contact eq 7.11. The capacitance may be computed in finite terms with elementary functions when $\omega=n \pi / m$ where $0<\omega \leq 2 \pi, m$ and $n$ positive integers.

The case, $\omega=\pi / m$ capacitance by eq 8.9 $\omega=(2 n-1) \pi / m$, for $1<n \leq 2 m$ by eq 8.10

$\omega=2 \pi$ (thin shell with aperture $2 \theta$ figure 1b) by eq 8.11 $\omega=2 \pi / m(m>1)$ by eq 8.12 $\omega=2 n \pi /(2 m-1),(1<n<2 m-1)$ by eq 8.13

Special cases,

$\omega=\pi / 2$ eq (8.14) and $\omega=3 \pi / 2$ (eq 8.15) $\omega=3 \pi / 2, \quad(\theta=\pi / 2)$ hemisphere (eq 8.16) $\omega=2 \pi,(\theta=\pi / 2)$ hemispherical bowl (eq $8.17)$

The potential due to grounded conductor in presence of a point-charge $M^{\prime}$ at any point $P^{\prime}\left(\alpha^{\prime}, 0\right)$ on the $x$-axis may be computed at any point $P(\alpha, \beta)$ by eq 4.9 which is

$$
V_{\omega}\left(\alpha, \beta ; \alpha^{\prime}, 0\right)=\frac{M^{\prime}}{c} \sin \left|\frac{\alpha^{\prime}-\theta}{2}\right| \sqrt{\frac{\cosh , \beta-\cos (\alpha-\theta)}{2}}\left[S_{\omega}\left(\alpha-\alpha^{\prime}, \beta\right)-S_{\omega}\left(\alpha+\alpha^{\prime}, \beta\right)\right]
$$

Since $S_{\omega}(\alpha, \beta)$ denotes $S_{\omega}(\alpha, \beta, 0)$, the second series (4.9) with $\beta^{\prime}=0$ may be used for these $S$-functions. This gives

$$
V_{\omega}\left(\alpha, \beta ; \alpha^{\prime}, 0\right)=\frac{8 M^{\prime}}{c \omega} \sin \left|\frac{\alpha^{\prime} \theta}{2}\right| \sqrt{\frac{\cosh \beta-\cos (\alpha-\theta)}{2}} \cdot \sum_{n=1}^{\infty} Q_{\frac{n \pi}{\omega}-\frac{1}{2}}(\cosh \beta) \sin \frac{n \pi \alpha}{\omega} \sin \frac{n \pi \alpha^{\prime}}{\omega},
$$

which converges for all points not on the $x$-axis, with exponential convergence factor $\exp \left(-\frac{n \pi}{\omega}+\frac{1}{2}\right) \beta$, so the first term $\mathrm{N}=1$, is the principal part when $\beta$ is large.

For computing $V$ on the $x$-axis, the $S$-functions in eq 10.1 may be expressed in terms of $G$-functions by use of eq 6.2. Then use of the series (6.7) gives

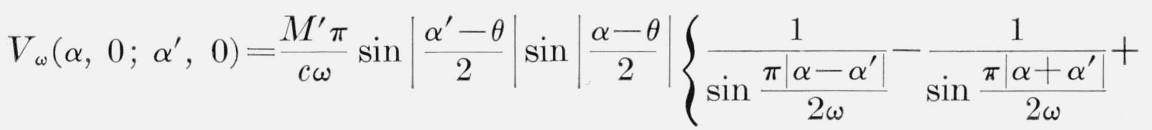

$$
\begin{aligned}
& \left.\frac{8}{\pi} \sum_{n=1}^{\infty} \sin \frac{n \pi \alpha}{\omega} \sin \frac{n \pi \alpha^{\prime}}{\omega}\left[\psi\left(n+\frac{1}{2}\right)-\psi\left(\frac{n \pi}{\omega}+\frac{1}{2}\right)+\log \frac{\pi}{\omega}\right]\right\} .
\end{aligned}
$$

This series could be reduced to one more rapidly convergent as in the transformation from eq 7.2 to 7.9 .

$$
\begin{aligned}
V_{\omega}(\alpha, \beta)= & V^{0}\left\{1-\sqrt{\frac{\cosh \beta \cos (\alpha-\theta)}{2}}\right. \\
& {\left.\left[S_{\omega}(\alpha-\theta, \beta)-S_{\omega}(\alpha+\theta, \beta)\right]\right\} . }
\end{aligned}
$$
due only to the conductor charged to a constant potential, eq 6.11 gives 
By use of the series (4.9) this becomes

$$
\begin{aligned}
V_{\omega}(\alpha, \beta)= & V^{0}-\frac{8 V^{0}}{\omega} \sqrt{\frac{\cosh \beta-\cos (\alpha-\theta)}{2}} . \\
& \sum_{n=1}^{\infty} Q_{\frac{n \pi}{\omega}-\frac{1}{2}}(\cosh \beta) \sin \frac{n \pi \theta}{\omega} \sin \frac{n \pi \alpha}{\omega} \cdot\left(10.2^{\prime}\right)
\end{aligned}
$$

On the $x$-axis this may be replaced by

$$
\begin{aligned}
V_{\omega}(\alpha, 0)= & V^{0}-\frac{\pi V^{0}}{\omega} \sin \left|\frac{\alpha-\theta}{2}\right|\left\{\frac{1}{\sin \frac{\pi|\alpha-\theta|}{\omega}}-\right. \\
& \frac{1}{\sin \frac{\pi(\alpha+\theta)}{\omega}+\frac{8}{\pi} \sum_{n=1}^{\infty} \sin \frac{n \pi \alpha}{\omega} \sin \frac{n \pi \theta}{\omega}} \\
& {\left.\left[\psi\left(n+\frac{1}{2}\right)-\psi\left(\frac{n \pi}{\omega}+\frac{1}{2}\right)+\log \frac{\pi}{\omega}\right]\right\} . }
\end{aligned}
$$

The potential at any point $P \alpha, \beta)$, due to the gounded conductor in presence of a circular linecharge $M^{\prime}$, with trace at any point $P^{\prime}\left(\alpha^{1}, \beta^{\prime}\right)$ is given by eq 5.1 and 5.2 where the $S$-functions may be computed by the series (4.9). In case $\omega=\pi / m$
( $m$ a positive integer) the $S$-functions are given in finite terms by eq 4.12 with $\omega=\pi$, that is,

$$
S_{\pi / m}\left(\alpha \pm \alpha^{\prime}, \beta, \beta^{\prime}\right)=\sum_{t=0}^{m-1} S_{\pi}\left(\alpha \pm \alpha^{\prime}+\frac{2 \pi t}{m}, \beta, \beta^{\prime}\right),
$$

where each $S_{\pi}$ function is given in terms of a complete elliptic integral in eq 2.32 .

In the case of a point-charge on the $x$-axis, the $S_{\omega}$-functions of eq 10.1 are given in finite terms when

$$
\omega=n \pi / m \text { where } n=1,2,3 \text {, or } 4 \text {, and } m
$$

is any positive integer. Similarly, the potential of the charged conductor alone is given by eq 10.2.

The expressions are found in eq 9.5, 9.6, 9.7, and 9.8 for $S_{2 \pi}(\alpha, \beta), S_{3 \pi}(\alpha, \beta)$ and $\mathrm{S}_{4 \pi}(\alpha, \beta)$. The last two cases, $n=3$ and $n=4$, involve elliptic functions. Using these in the general formula (eq 4.12) with $\omega=n \pi$ and $\beta^{\prime}=0$ gires

$$
S_{n \pi / m}(\alpha, \beta)=\sum_{t=0}^{m-1} S_{n \pi}\left(\alpha+\frac{2 n \pi t}{m}, \beta\right) .
$$

Washington, February 8, 1949. 